Invited paper presented at the NATO Advanced Study Institute on "Multicomponent and Multilayered Thin Films for Advanced Microtechnologies. Techniques, Fundamentals and Devices". Bad Windsheim. Fed. Rep. Germany. Sept. 22- Oct. 6, 1992

\title{
ION BEAM-BASED CHARACTERIZATION OF MULTICOMPONENT OXIDE THIN FILMS AND THIN FILM LAYERED STRUCTURES
}

\author{
ALAN R. KRAUSS, MUKUND RANGASWAMY, YUPING LIN, DIETER M. GRUEN, \\ Materials Science and Chemistry Divisions, Argonne National Laboratory. Argonne IL 60439 \\ J. ALBERT SCHULTZ, Ionwerks Incorporated, Houston, TX \\ HOWARD K. SCHMIDT, Schmidt Instruments Incorporated, Houston, TX \\ and R. P. H. CHANG, Materials Science Department. Northwestern University, Evanston IL
}

The fabrication of thin film layered structures of multi-component materials such as high temperature superconductors, ferro-electric and electro-optic materials, and alloy semiconductors, and the development of hybrid technologies incorporating some or all of these materials into a single device requires a detailed understanding of film growth and interface properties. Some of the relevant interfaces are within a few $\AA$ of the surface, while others may be at a depth of several $\mu \mathrm{m}$. Interfaces may be either atomically abrupt or be spread over a significant depth, with no clearly identifiable transition.

For materials such as the High Temperature Superconductors (HTSC's), the superconducting coherence length is extremely short (5-15 $\AA$ ), and the fabrication of reliable devices utilizing these materials will require control of film properties at extremely sharp interfaces; It will be necessary to verify the integrity of thin layers and layered structure devices over thicknesses comparable to the atomic layer spacing. Analytical techniques which probe the first 1-2 atomic layers are therefore necessary for in-situ characterization of relevant thin film growth processes. However, most surface-analytical techniques are sensitive to a region within 10-40 $\AA$ of the surface. Additionally, they are physically incompatible with existing thin film deposition technologies and are typically restricted to ultra high vacuum conditions, whereas the growth of multi-component oxide or nitride films is usually carried out in an ambient partial pressure of oxygen or nitrogen up to several hundred mtorr.

A review of ion beam-based analytical methods for the characterization of thin film and multi-layered thin film structures incorporating layers of multicomponent oxides will be presented. Particular attention will be paid to the use of time-of-flight techniques based on the use of $1-15 \mathrm{keV}$ ion beams which show potential for use as non-destructive, real-time, in-situ surface diagnostics for the growth of multicomponent metal and metal oxide thin films.

\subsection{Introduction}

\subsection{IN-SITU MONITORING OF THIN FILM DEPOSITION PROCESSES}

Thin film deposition methods such as ion beam sputtering which can be applied to a wide range of materials are ideally suited to the production of multicomponent thin films and layered structures as part of a single automated process. One such deposition process is described elsewhere in these proceedings [1]. Films produced by such methods may incorporate materials representing semiconducting, ferroelectric, optoelectronic, magnetic and high temperature superconducting technologies, or a combination of such materials in a single hybrid device. As more and more complex thin film structures are produced, it becomes increasingly more important to be able to monitor the deposition process in-situ. This is particularly so when dealing with oxide and nitride films in which the phase produced is a highly sensitive function of the growth conditions. However, it is precisely these materials which present the greatest challenge to in-situ surface analysis. It has for example been found that the best $\mathrm{YBa}_{2} \mathrm{Cu}_{3} \mathrm{O}_{7-x}$ thin films have critical current densities approximately $100 \mathrm{x}$ higher than bulk polycrystalline material. A connection has been made [2] between the high critical current density observed in some HTSC thin films and a high density of screw dislocations believed to be associated with island growth during the early stages of film

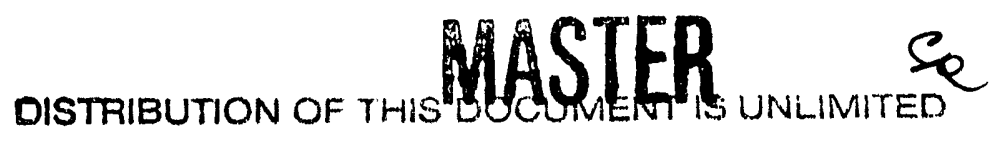




\section{DISCLAIMER}

This report was prepared as an account of work sponsored by an agency of the United States Government. Neither the United States Government nor any agency thereof, nor any of their employees, makes any warranty, express or implied, or assumes any legal liability or responsibility for the accuracy, completeness, or usefulness of any information, apparatus, product, or process disclosed, or represents that its use would not infringe privately owned rights. Reference herein to any specific commercial product, process, or service by trade name, trademark, manufacturer, or otherwise does not necessarily constitute or imply its endorsement, recommendation, or favoring by the United States Government or any agency thereof. The views and opinions of authors expressed herein do not necessarily state or reflect those of the United States Government or any agency thereof. 
nucleation. The ability to characterize the early stages of film formation would provide a means of unambiguously identifying the growth processes which lead to high $\mathrm{J}_{\mathrm{c}}$ values, and help to assure the attainment of those growth conditions during device fabrication.

All surface characterization schemes involve directing ions, electrons, or photons onto the sample, and detecting the ejected ions, electrons, or photons resulting from a number of different physical processes within the solid. For example, X-ray Photoelectron Spectroscopy (XPS) involves the impact of an incident $x$-ray beam on the analyzed material to create photoelectrons. The kinetic energy of these photoelectrons is uniquely related to the electron energy levels of the atoms within the solid and can therefore be used to identify the atoms. The surface sensitivity of XPS is due to the fact that only photoelectrons generated in a shallow layer near the surface can escape to the vacuum to be detected. For electrons with a kinetic energy $\leq 1 \mathrm{keV}$, the depth of analysis varies from approximately 10 to $40 \AA$. However, if there is an ambient gas pressure associated with the deposition process, the short electron mean free path becomes a disadvantage since the electron must travel distances on the order of 0.5 meters through an energy analyzer before it reaches the detector. Techniques such as Auger Electron Spectroscopy (AES), Ultraviolet Photoelectron Spectroscopy (UPS), Low Energy Electron Diffraction (LEED), and XPS typically require high to ultra-high vacuum $\left(<10^{-8}\right.$ torr) in the region surrounding the sample.

There is an additional practical constraint on most surface analytical methods in regard to in-situ monitoring of thin film deposition in that the analysis equipment must not intrude in the area occupied by the deposition equipment. In order to obtain relatively high data acquisition rates, most surface analytical equipment requires the positioning of large instrumentation very close to the sample surface. The number of analytical techniques which are suitable for in-situ characterization of surface processes during thin film growth is therefore very limited. There are several techniques which utilize relatively collimated beams and therefore do not interfere with the deposition process. Reflection High Energy Electron Diffraction (RHEED) utilizes elastically scattered electrons with a kinetic energy in the range of $20 \mathrm{keV}$. This energy is high enough to provide a reasonably long electron mean free path. RHEED is therefore widely used in molecular beam epitaxy (MBE) systems where the ambient pressure during deposition is relatively low. RHEED provides a measure of the lattice spacing in the direction normal to the substrate, but provides no chemical identification and no information on short-range phenomena such as pinhole formation.

Techniques such as $\mathrm{x}$-ray diffraction (XRD), $\mathrm{x}$-ray fluorescence spectroscopy (XFS), Ion-Induced X-ray spectroscopy (IIX), Rutherford backscattering spectroscopy (RBS) and Elastic Recoil Detection (ERD) can be used for in situ characterization of the overall properties of a film, but except for grazing incidence geometries, they sample the material to a depth on the order of 0.5 to $1 \mu \mathrm{m}$. In order to characterize the processes occurring at the surface of a growing film, it is necessary to probe the first few atomic layers, and in principle to identify the uppermost monolayer where the growth occurs.

\subsection{Pulsed Ion Beam Surface Characterization}

Low energy $(1-15 \mathrm{keV})$ pulsed ion beam surface-analytical techniques, possess the ability to provide a remarkably wide range of information directly relevant to the growth of multi-component semiconductor, metal and metal oxide thin films and layered structures. The range of information obtainable by Direct Recoil Spectroscopy (DRS) and Ion Scattering Spectroscopy (ISS) probably exceeds that obtainable by any other surface analytical technique. The information available with these methods includes the surface composition, atomic structure of the first few monolayers, lattice defect density, trace element analysis and phonon characteristics. Much of this data may be obtained on samples which are at ambient gas pressures up to one torr, i.e. 6-8 orders of magnitude higher than any other analysis technique which provides a comparable degree of surface specificity. This capability makes pulsed ion beam analysis ideal for real-time, in-situ characterization of thin film growth processes. We discuss here some of the materials properties which may be measured by Direct Recoil Spectroscopy, (DRS), Ion Scattering Spectroscopy (ISS) and Mass Spectroscopy 
of Recoiled Ions (MSRI), and describe a physical implementation of the pulsed ion beam analysis technique which requires very low beam dose, provides real-time data acquisition, does not interfere physically with thin film deposition equipment, is compatible with high ambient pressure operation, and is therefore suitable as a real-time probe of thin film deposition. DRS/ISS in particular, will be shown to be non-destructive, real-time, in-situ surface diagnostics, suitable for monitoring the growth of multicomponent metal and metal oxide thin films.

\section{1 THEORY}

Ion Scattering Spectroscopy was demonstrated to be a useful means of thin film analysis by Smith in 1971 and by McKinney and Frankenthal in 1973 [3.4]. The McKinney study used ISS as a means of characterizing 20-30 $\AA$ thick passivation layers on $\mathrm{Fe}-\mathrm{Cr}$ alloys. However, ISS has not been widely used for this purpose for a number of reasons: Early ISS instruments required a very high ion beam dose, sometimes exceeding the number of atoms at the surface of the film being analyzed in order to produce usable spectra. In order to achieve this dose, data acquisition times could be longer than the film deposition time. Because of the extreme surface sensitivity of the ISS technique, it is commonly perceived as suitable only for use in ultra-high vacuum and therefore incompatible with many thin film deposition methods. Additionally, most ISS analyzers including even currently marketed commercial instruments are geometrically incompatible with most deposition processes. Modern Time-of-Flight (ToF) ISS instruments are either not in fact subject to most of these limitations, or can be modified to correct these difficulties. However, ToF instruments are still relatively rare, existing only as custom-built units in a few laboratories.

The method of ion beam surface analysis consists of directing an ion beam of mass $\mathbf{M}_{1}$, kinetic energy $E_{0}$ at the surface, consisting of atoms with mass $M_{2}$, and detecting either the backscattered primary particles at energy $\mathrm{E}_{1}$ (ISS), or the direct recoil-sputtered surface atoms (DRS) with energy $E_{2}$, as shown in Fig. 1.

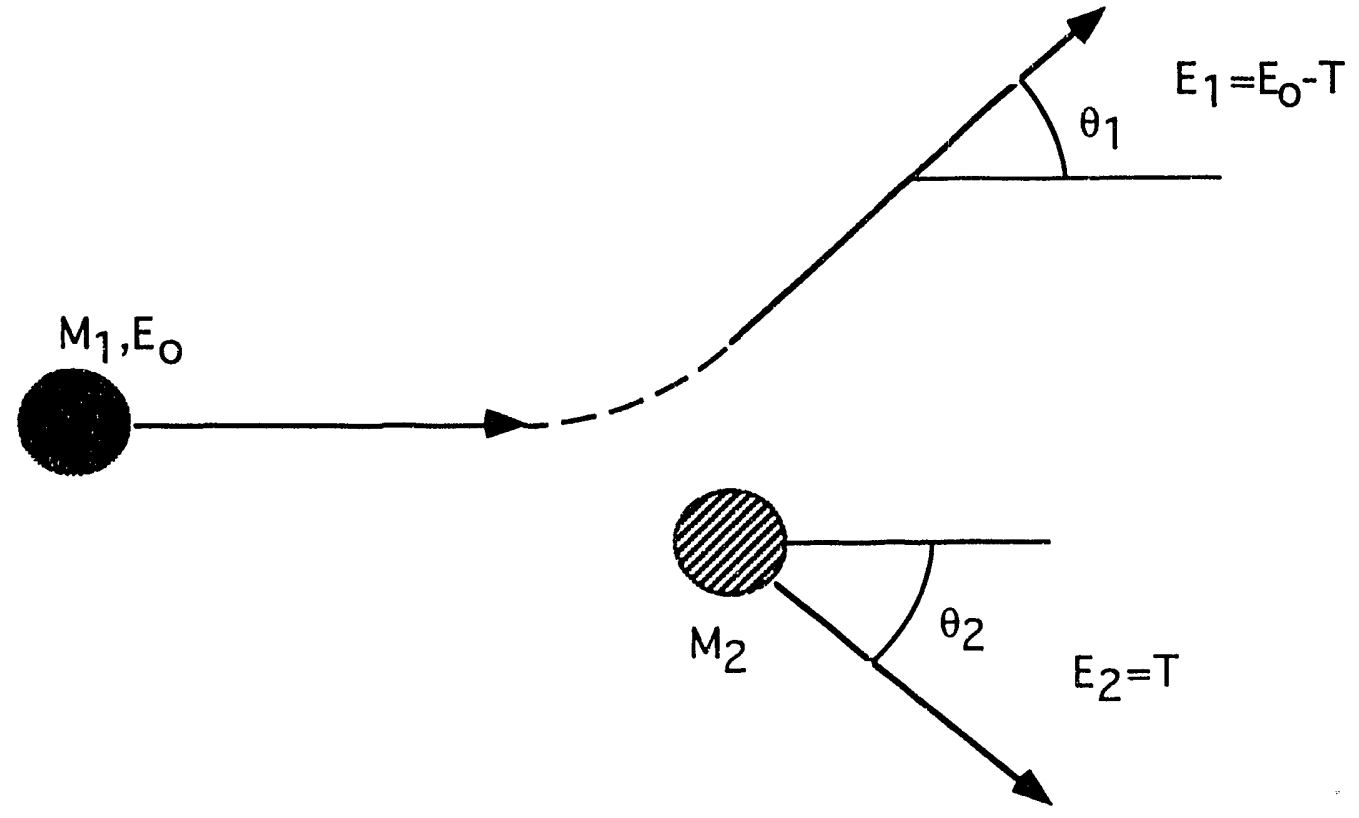

Fig. 1. Collision geometry for an ion, with mass $M_{1}$, kinetic energy $E_{1}$ (black circle) incident on a surface atom with mass $\mathrm{M}_{2}$ (hatched circle). $T$ is the kinetic energy transferred during the collision.

For primary ions in the approximate range $1-100 \mathrm{keV}$, the primary ion-target atom collisions are adequately described by two-body elastic collision dynamics. The kinetic energy, $E_{1}$ of the scattered primary is then given by 


$$
\frac{E_{1}}{E_{o}}=(1+\alpha)^{-2}\left[\cos \theta_{1} \pm\left(\alpha^{2}-\sin ^{2} \theta_{1}\right)^{1 / 2}\right]^{2}
$$

provided $M_{2}>M_{1}$. The kinetic energy $E_{2}$ of the recoil-sputtered surface atom is

$$
\frac{E_{2}}{E_{o}}=4 \alpha(1+\alpha)^{-2} \cos ^{2} \theta_{2}
$$

where $\alpha=M_{2} / M_{1}$ and $\theta_{1}$ and $\theta_{2}$ are the scattering and recoil angles respectively. In addition to the single collision events represented by eqs. 1 and 2 , more complicated multiple collision events sometimes give rise to relatively sharp peaks as well. These often occur at energies for which no single scattering event is possible and can usually be identified.

In order to monitor thin film deposition, it is necessary to obtain data over periods of time which are short compared with the time required for the thin film deposition process, using ion beam doses which result in negligible sputtering or other modification of the surface being studied. Since there are roughly $10^{15}$ atoms $/ \mathrm{cm}^{2}$ at the surface of a typical solid, a non-damaging dose may be taken as approximately $10^{13} \mathrm{ions} / \mathrm{cm}^{2}$. The kinetic energies of the scattered primary and direct recoil atoms are typically measured either by using an electrostatic energy analyzer (ESA) [3-5] with a continuous beam, or by pulsing the beam and measuring the time required for the scattered/sputtered particles to reach the detector after the ion beam strikes the sample $[6,7]$. The ESA detects only ions, which typically constitute between 0.1 and $1 \%$ of the total flux leaving the sample. Moreover, at any given time, the ESA is set to transmit only a very narrow range of kinetic energies, and the spectrum is obtained by scanning this energy window.

The ToF-ISS method consists of directing a pulsed beam of energetic ions onto the surface and measuring the time at which the scattered primary particles, most of which are scattered as neutral atoms, arrive at the detector. By placing the detector either off-axis or in line of sight with the sample, either the scattered ions or neutrals may be selectively detected. If the scattered neutrals are detected, the method has a depth sensitivity of 2-3 atomic layers. However, since the neutralization probability for an ion penetrating more than one monolayer into the solid is nearly unity, the detection of the scattered ion species provides a sensitivity only to the uppermost atomic layer of the solid $[3,5,6,8]$. This surface specificity is unique among surface analytical techniques. Consequently, detection of the scattered ions provides an ideal method for the characterization of sharp interfaces. The ToF scheme, which independently detects backscattered ions and neutrals with kinetic energies corresponding to all $M_{2}$ values simultaneously, gains 3-4 orders of magnitude in terms of the reduction in data acquisition time and ion beam dose necessary to obtain data compared with the ESA. Low beam dose and rapid data acquisition are key requirements for real-time, low damage in situ analysis of thin film growth.

The most obvious application of ISS for thin film analysis is the determination of the surface composition. Assuming that all atoms within a depth $\delta x$ are exposed to the same incident ion flux, quantitative determination of the composition depends on knowing the differential scattering cross section, $d \sigma / d \Omega$ and, for the ion signal, the probability that the scattered will not be neutralized, $\mathrm{P}^{+}$. The scattered ion signal is given by:

$$
\mathrm{I}^{+}=\mathrm{I}_{0} \Omega \text { T C } \mathrm{C}_{\mathrm{S}} \delta \mathrm{x}(\mathrm{d} \sigma / \mathrm{d} \Omega) \mathrm{P}^{+}
$$

where $I_{O}$ is the incident ion flux, $\Omega$ is the solid angle subtended by the analyzer, $T$ is the transmission of the analyzer, and $C_{S}$ is the surface concentration in a region of thickness $\delta x$. For primary ion kinetic energies approximately $1 \mathrm{keV}$ or greater, the differential cross section is determined by two-body collision processes, and the scattering intensity is proportional to the number of atoms exposed to the beam. The cross section may be determined using the 
theory of Lindhard, Scharff and Schiott [9-11] with e.g. a Thomas-Fermi potential using Moliere's approximation [12] to simplify the calculation.

The charge exchange probability for an ion traversing a distance $\underline{\mathrm{ds}}$ in time increment dt is given by [13]:

$$
\mathrm{P}_{\mathrm{n}}=\mathrm{A} \exp (-\mathrm{as}) \mathrm{dt}
$$

and $\mathrm{V}=\mathrm{ds} / \mathrm{dt}$ is the instantaneous primary ion velocity, $\mathrm{t}$ is time, and $\underline{a}$ is the thickness of the near-surface region in which charge exchange occurs. By integrating over the incoming and outgoing ion trajectory, the probability of scattering without neutralization is found to be

$$
\mathrm{P}^{+}=\exp -\left\{\int_{s_{1}}\left(\mathrm{~A} / \mathrm{V}^{1} \perp\right) \exp (-\mathrm{as}) \mathrm{ds}+\int_{\Omega_{2}}\left(\mathrm{~A} / \mathrm{V}^{2} \perp\right) \exp (\text {-as }) \mathrm{ds}\right\}
$$

where $\underline{A}$ represents an electron tunneling frequency, $\underline{\mathbf{s}} \underline{\underline{p}}$ and $\underline{\mathbf{s}}$ are the incoming and outgoing trajectories, and $\mathrm{V}_{\perp}^{1}$ and $\mathrm{V}_{\perp}^{2}$ are the normal component of the incoming and outgoing velocities respectively. Calculation of the ion survival rate is a non-trivial problem, and the results are strongly influenced by the chemical environment of the surface atoms. However, the magnitude of this so-called "matrix effect" depends exponentially on the ion velocity, and is consequently much smaller for relatively energetic scattered primary ions or direct recoil ions than it is for the much slower ions associated with Secondary Ion Mass Spectroscopy (SIMS).

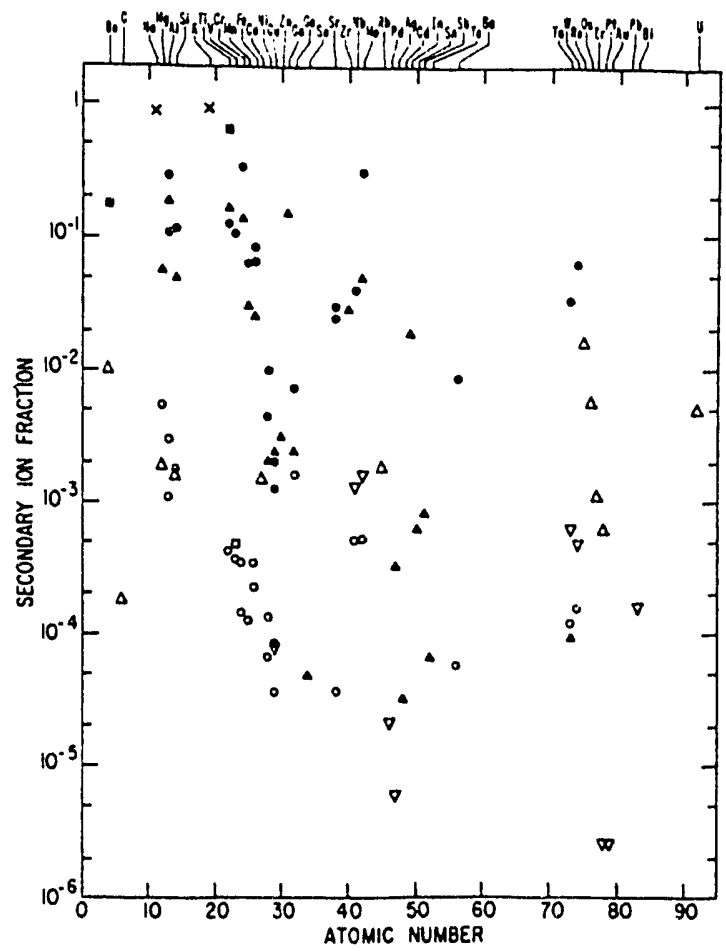

Fig. 2 Plot of the charge fraction for atoms sputtered from a variety of clean (open circles) and oxidized (filled circles) elements. (after Krauss \& Krohn, 1980)

In general, the scattered ion and direct ion recoil intensities deviate from the values corresponding to the calculated cross sections by a factor of $2-4 \times[14,15]$ from one surface atom species to another, while the SIMS intensities vary by as much as 5 orders of magnitude as shown in Fig. 2 [16]. For a given surface species, the ion component of the ISS and DRS 
intensities vary by $2-4 x$ as the degree of oxidation is changed, while the SIMS signal varies by 2-3 orders of magnitude. For example, the removal of an oxide layer on copper by ion beam sputtering results in a two-fold increase in the ion fraction of the ISS signal, but a 2 order of magnitude decrease in the $\mathrm{Cu}^{+}$SIMS signal as shown in Fig. 3 [17]. Alkali metals which dominate SIMS spectra even when they are present as trace impurities represent an extreme example in that they are typically not even seen in ISS spectra [17]. This occurs since the secondary ion fraction for alkali metals present as trace impurities is in the range $50-100 \%$, whereas for most oxygen-free metallic species, the secondary ion fraction is $10^{-2}$. $10^{-5}$ and is largely independent of the primary ion energy.

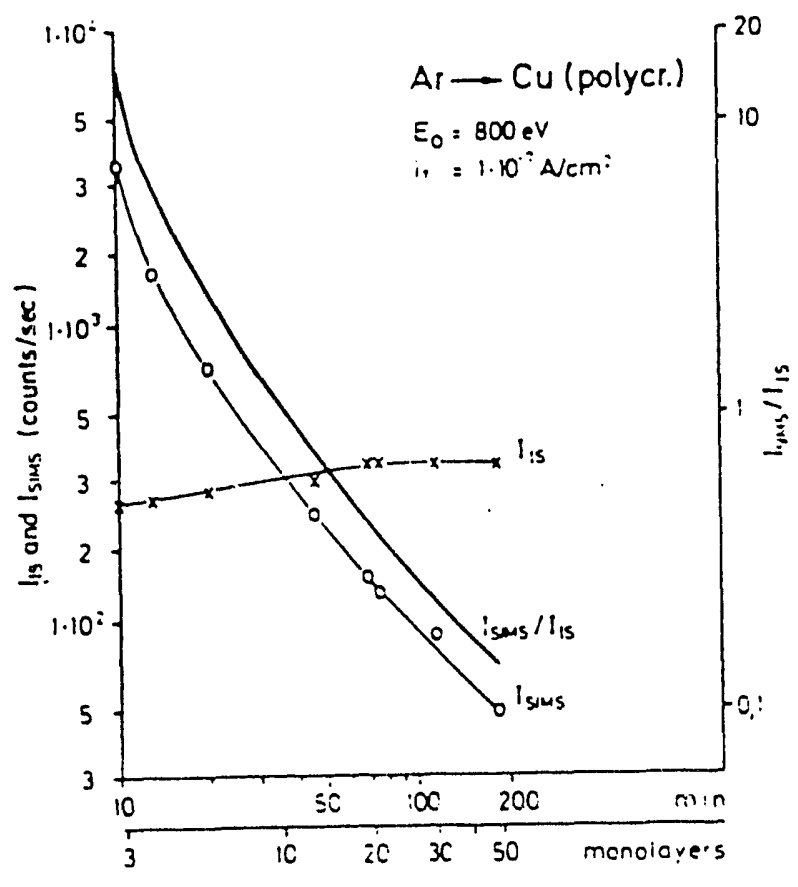

Fig. 3 Detected ion signals corresponding to ISS and SIMS for an oxidized $\mathrm{Cu}$ surface cleaned by ion beam sputtering (After Grundner et. al., 1974)

The absolute magnitude of the ion fraction observed in DRS and ISS is much larger, but somewhat more dependent on the primary beam energy than is the case for SIMS. For example, for 2-10 keV Ar${ }^{+}$incident on $\mathrm{SiO}_{2}$ and $\mathrm{Mg}(\mathrm{OH})_{2}$, the ion component of the DRS signal ranges from 30 to $46 \%$, and for $5-10 \mathrm{keV} \mathrm{Ar+}$ incident on $\mathrm{C}$ it varies from 4-10\%[18]. The positive ion fraction for He scattering from $\mathrm{Au}$ and $\mathrm{W}$ at $135^{\circ}$ ranges from $9-11 \%$ for primary ion energies ranging from 5-16 keV [19]. For some combinations of primary ion and target elements such as $\mathrm{He}^{+}$incident on $\mathrm{Pb}$, In and $\mathrm{Sn}$, oscillations in the ion component of the ISS signal with kinetic energy have been observed [20-22]. These oscillations have been attributed to a quasi-resonant charge exchange process in which the energy levels of the outer shell electrons of the sample are close to the He 1s state. Methods have been proposed to exploit this effect as a means of characterizing the chemical state of the surface atoms [23] but there seems to be little recent work in this area.

Therefore, with some qualifications, measurement of the ion component of the ISS signal constitutes a reasonably reliable means of measuring the surface composition a solid and of profiling near-surface interfaces with a very high degree of depth resolution. An example is shown in Fig. 4 [4], illustrating spectra of a passivation layer on a $\mathrm{Fe}-\mathrm{Cr}$ alloy, taken after various intervals of ion beam sputtering. The four spectra represent the composition at the surface, and at depths of 10,20 and $30 \AA$, respectively. Since the data produced by ISS is extremely surface specific, it is possible to observe significant variation in 
the $\mathrm{Cr}$ concentration over a range which is comparable with the mean sampling depth of XPS and AES. It should be noted however, that since an ESA was used, the analysis could not done during formation of the passivation layer, and that the data acquisition itself required a beam dose of $\sim 10^{15}$ ions and consumed 1-2 monolayers/scan, even for a relatively limited mass range. In order to obtain even this degree of sensitivity, it was necessary to collect ions scattered into a solid angle which was large enough to compromise mass resolution between $\mathrm{Fe}$ and $\mathrm{Cr}$.

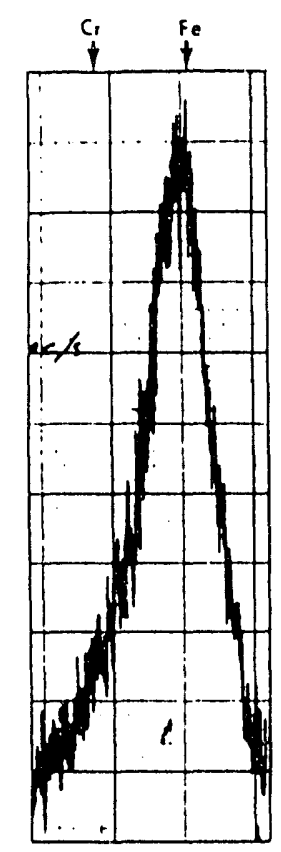

OUTER SURFACE

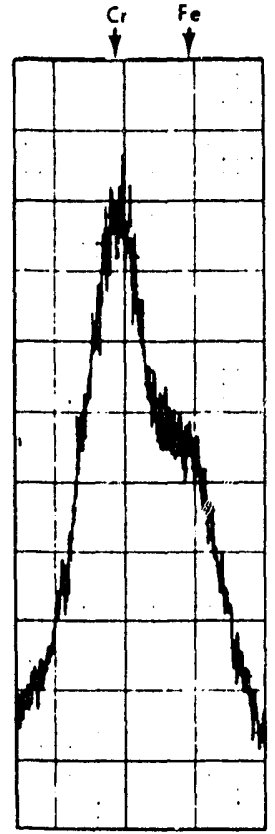

$\sim 10 \AA$ \& REMOVED

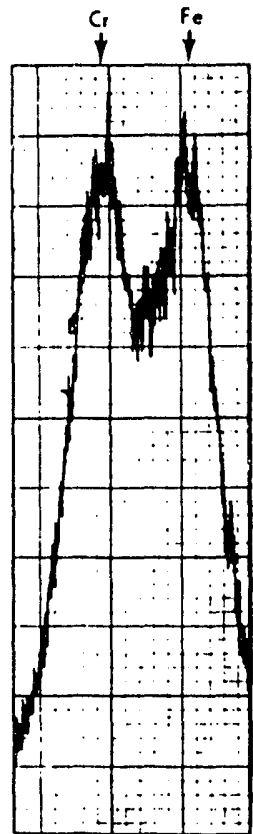

$\sim 20 \AA ̊ 丿$ REMOVED

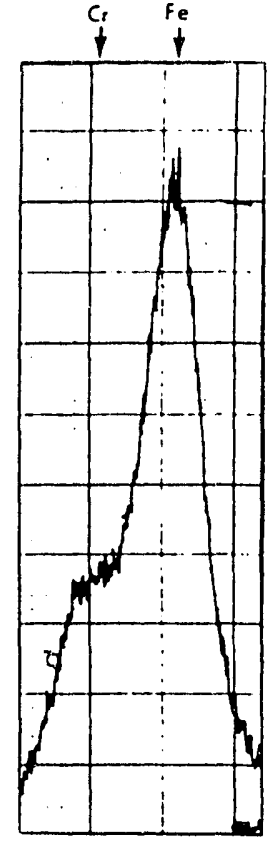

$\sim 30 \AA$ A REMOVEO

Fig. 4 ISS spectrum of stainless steel taken with $127^{\circ}$ electrostatic analyzer (after McKinney 1973)

In order to increase the sensitivity, electrostatic analyzer ISS systems sometimes use an alkali metal beam [24-30]. As suggested in Fig. 2, alkali metal atoms scatter predominantly as ions. It is therefore possible to use an electrostatic analyzer with a beam dose which is reduced by $\sim 100 \mathrm{x}$ compared with an inert gas ion beam. Aside from the increase in signal intensity, the alkali ion beam produces a broader peak, and a pronounced low energy tail, both resulting from multiple collisions involving subsurface atoms. The angular intensity variation of the alkali ion signal shows more structure than the inert gas data. The additional structure can be interpreted in terms of single collisions involving either first or second layer atoms or double collisions, usually involving one first layer and one second layer atom. Complete characterization usually requires postulating different models of the near-surface structure, using computer methods to simulate the corresponding spectra [27].

An even larger increase in sensitivity can be obtained by detecting backscattered neutral inert gas ions [31]. Detection of energetic neutrals requires the use of the somewhat more complicated time-of-flight methods, but because all masses are sampled simultaneously, the ion beam dose is even lower than that required for alkali metal beam scattering. Additionally, the inert gas beam ions which are implanted into the sample are much less harmful to most film properties than the alkali metals. The surface specificity of backscattered neutrals is not quite as great as that of the backscattered ion signal. However, as will be shown below, the use of backscattered neutrals makes it possible to detect atoms in the 2 nd and 3 rd atomic layers, and to quantitatively determine their position in the crystal lattice. 


\subsection{APPLICATION}

2.2.1 Elemental Abundances. It is possible to utilize the surface-specificity of ion scattering spectroscopy (both ion and neutral signals) to obtain highly detailed information about the structure and defect properties of the first few atomic layers of a solid. [7,32-38]. Consider for example, a solid in which the first layer of atoms are of species A (open circles in Fig. 2), and the second layer consists of species B (filled circles). As shown in Fig. 5, the angle at which the primary ions are scattered depends on the distance between the initial primary ion trajectory and the center of the target atoms. There is a shadow cone into which no primary ions are scattered, and an enhancement of the scattering intensity on the surface of the shadow cone region. If the B atoms in the second atomic layer lie within the shadow cone of the first layer A atoms as shown in Fig. 5a, they do not contribute to primary ion scattering. There is an angle of incidence for which the second layer atoms lie on the surface of the shadow cone of the first layer atoms as shown in Fig. 5b, For this critical angle of incidence, the second layer atoms intercept an enhanced primary ion flux and produce an enhanced scattering signal. If the second layer atoms lie outside the shadow cones of the first layer atoms, they receive the same primary ton flux as the first layer atoms, as shown in Fig. 5c, and the signal intensity may be used to quantify the relative concentration of $A$ atoms and $B$ atoms. It has been found that surface roughness results in a decrease in the absolute intensity of the scattering peaks. However [39] it has been found that the relative intensities of the surface components remain unchanged, and ISS therefore provides valid composition ratios for rough "real-world" samples as well as polished laboratory samples.

a

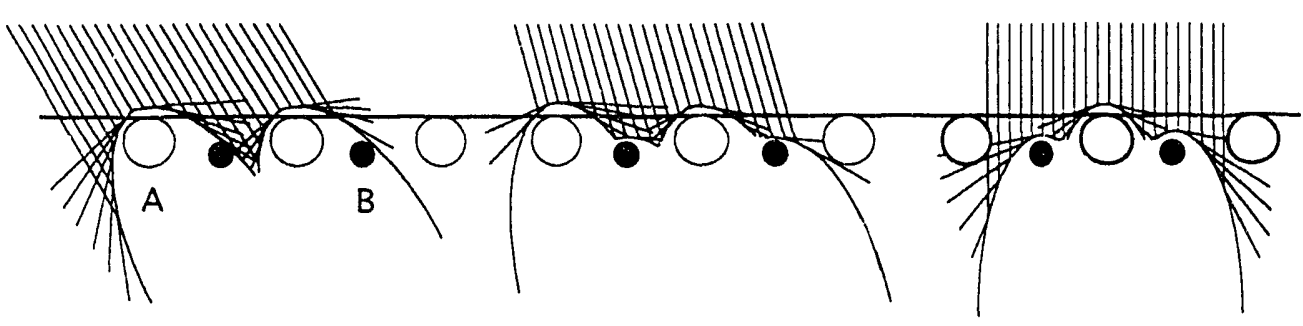

Fig. 5. Illustration of the shadowing phenomenon. (a) Atom B (filled circles) is completely inside the shadow cone of atom A (open circles) and does not give rise to an ISS signal. (b) Atom B is on the edge of the shadow cone of atom $A$ and is receiving an enhanced primary ion flux. (c) Atom $B$ is completely outside the shadow cone of atom $\mathrm{A}$ and receives the same primary ion flux as $\mathrm{A}$.

2.2.2 Surface Crystallography. An example of the shadowing phenomenon is shown in Fig. 6 for a monolayer of Au adsorbed on Si (111) [40]. Since the gold is in the uppermost atomic layer, the signal intensity for backscattered primary ions is almost independent of the ion beam angle of incidence. It can therefore be immediately determined that the Au film is not more than one monolayer thick. The Si atoms however, reside entirely in the second and deeper layers, and for certain angles of incidence, they are shadowed by the adsorbed $\mathrm{Au}$ layer, resulting in oscillations in the $\mathrm{Si}$ scattering intensity as a function of the angle of incidence.

If we consider the case of grazing ion beam incidence on the surface shown in Fig. 5 , there is a critical angle for the surface atom species as well as for the second layer atoms. This is illustrated in Fig. 7. For near-grazing incidence (Fig. 7a) the surface atoms lie in each other's shadow cone, and the scattered signal intensity is nearly zero. At somewhat larger angles (Fig. 7b), each surface atom lies on its neighbor's shadow cone, and a strong signal is seen. At still larger angles of incidence (Fig. 7c), the scattering intensity for the surface atoms becomes independent of the angle of incidence. 


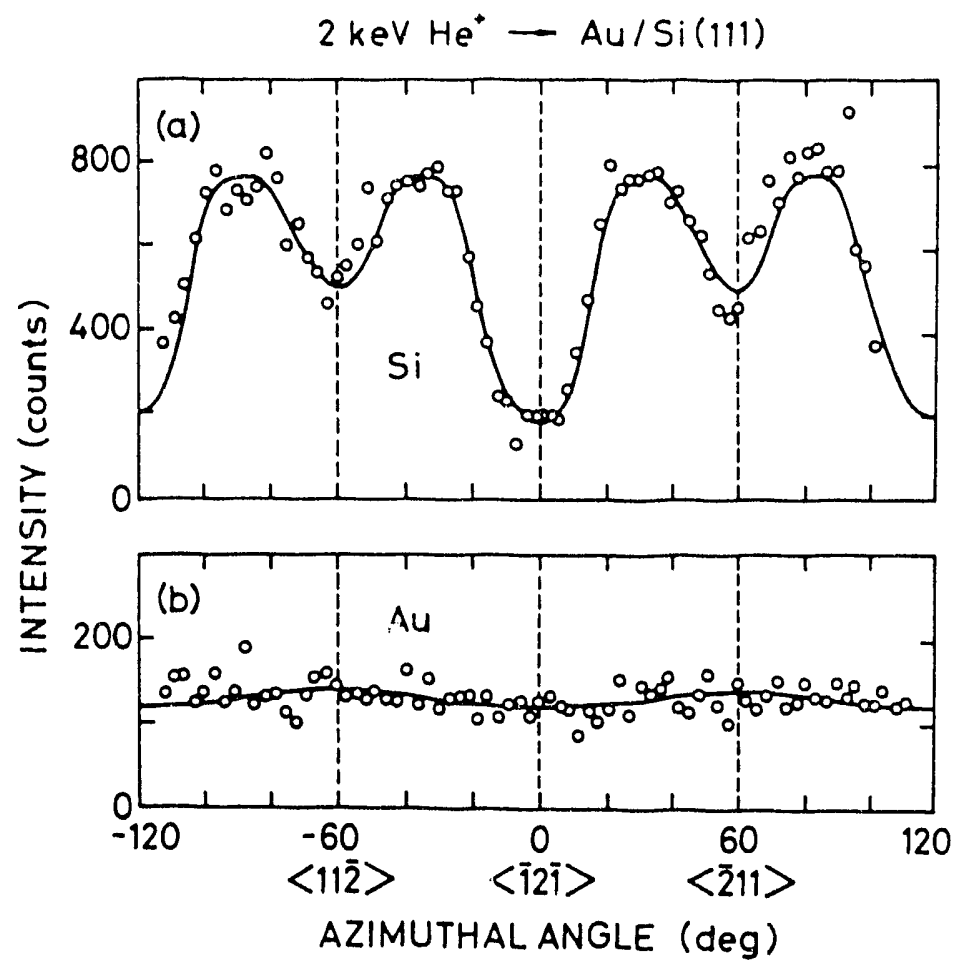

Fig. 6. Au/Si Angular variation of the Au and Si ISS signal intensity for an Au monolayer adsorbed on Si (111) (after Katayama et. al. 1988).

a

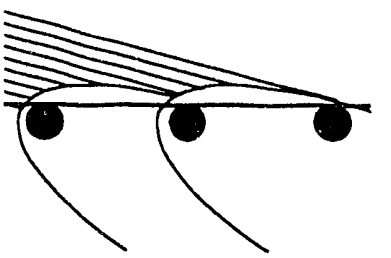

b

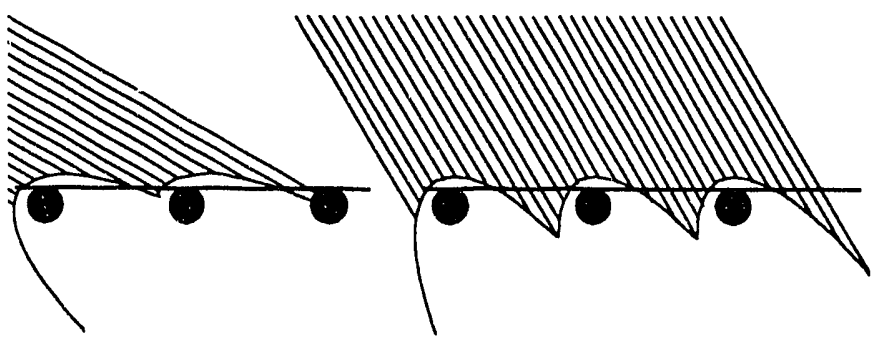

Fig. 9. Self-shadowing of surface atoms at oblique angles of primary ion incidence.

By mapping the intensity of specific peaks of a multicomponent material, as a function of both azimuthal and polar angles, it is possible [41] to construct an ion beam crystallograph, showing a psuedo-three dimensional image of the atoms in the first few atomic layers. Data for As/Si(001) are shown in Fig. 8. The As image, shown in Fig. 8a exhibits a strong intensity variation for polar angles close to grazing incidence, but very little variation with azimuthal angle and for polar angles greater than $\sim 6$ degrees. In accord with the preceding paragraph, it is therefore immediately obvious that the As exists as a Inonolayer, with no depth-dependent structure. The Si substrate however, has a well-defined 3-dimensional structure, and as shown in Fig. 8b, such features as the atomic alignment and locations of open channels in specific crystallographic directions are clearly seen.

\subsubsection{VIBRATIONAL ENERGY}

If the atoms on the surface are absolutely stationary, the variation in signal intensity due to the shadowing effect would be arbitrarily sharp. In practice however, the atoms are not stationary and the sharpness of the increase in the $B$ atom signal as the angle of incidence is varied through the critical angle $\left(\psi_{\mathrm{C}}\right)$ where the edge of the $\mathrm{A}$ atom shadow cone starts to intercept atom $B$, is a measure of the mean vibrational energy of the surface atoms. An 
example is shown in Fig. 9 for the TiC (111) surface. The Ti ICISS signal is shown as at tunction of angle of incidence for 3 different azimuths. The mean displacement from the stationary atom positions maty be calculated by assuming a given atomic displatcement distance. calculating the critical angle and summing the restilts for a range of displacements weighted by the probahility of the given displacement using a Gaussian distribution function. The points in Fig. 9 represent experimental data for the three azimuths. and the solid lines represent the best fit results for the computer simulations. The mean square atomic displacements measured for the three azimuths are shown in the inset. [43-52]

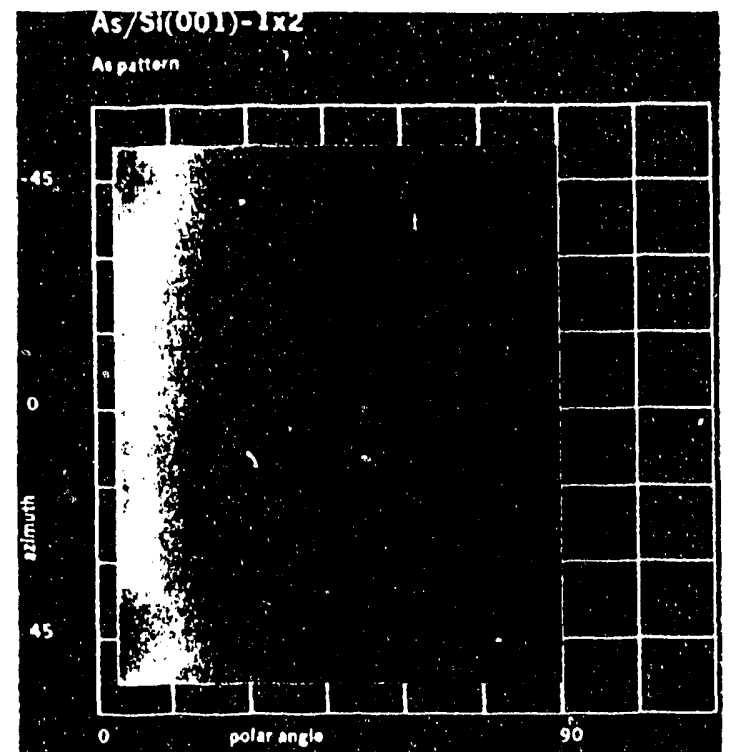

(a)

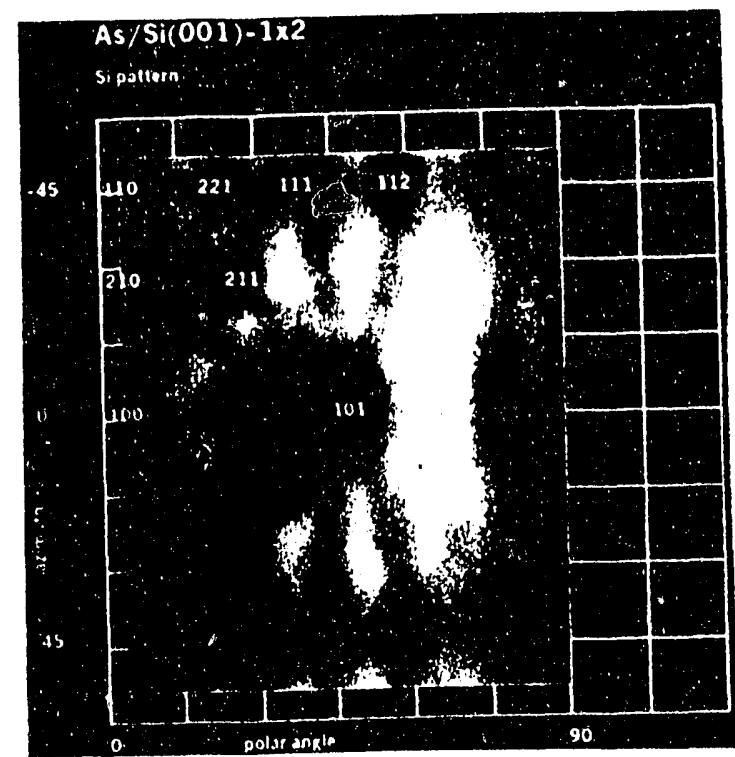

(b)

Fig. 8. Ion beam crystallograph for As/Si (001) - (1 $\times 2)$ produced by (a) monitoring the As ISS signal intensity and (b) by monitoring the Si ISS signal as the polar and azimuthal angles are scanned. (After Niehus \& Spitzl. 1991)

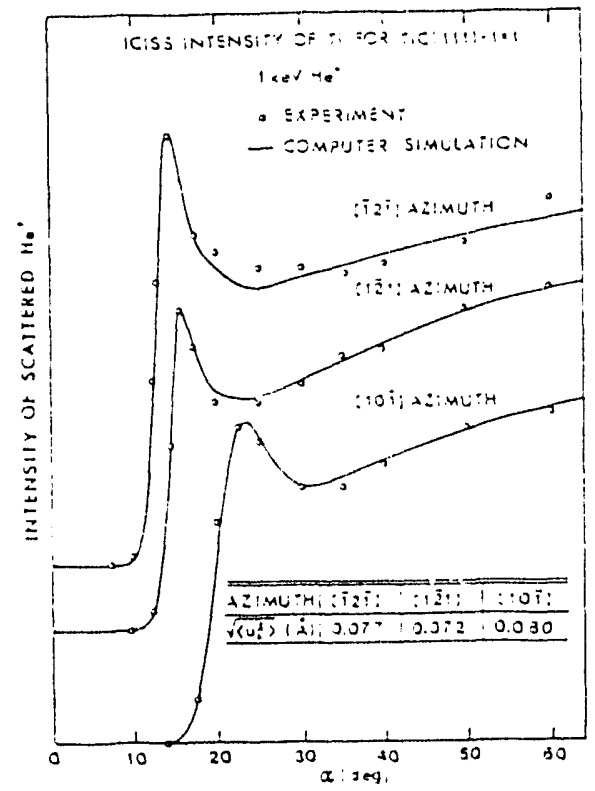

Fig. 9. Circles: intensity of He- ions scattered from Ti atoms at the TiC (111)-1xl surfilce an a function of ion incidence angle $\alpha$. (measured from the surface). The solid curves are the best-fit computer simulation results. The table shows the values of the root mean square displacement perpendiculiar to the surface, in Angstroms. (. Aiter Soulta et. al., 1983) 
2.2.4 Surface Atomic Structure. The shadow cone is defined as the envelope of the points of closest approach for ions with a range of impact parameters $r$. The shape of the shadow cone is described in terms of its radius $\underline{r}$ as a function of the distance $\underline{R}$ along the axis of the shadow cone from the scattering center. $\underline{C}$ is defined as the hypotenuse of the right triangle formed by $\mathrm{R}=\mathrm{C} \sin \left(\Psi_{\mathrm{C}}\right)$ and $\mathrm{r}=\mathrm{C} \cos \left(\psi_{\mathrm{C}}\right)_{\text {. }}$ The relationship between $\underline{\mathrm{R}}$ and $\underline{\mathrm{r}}$ is determined by the interatomic potential. At the angle of incidence $\psi_{c}$ at which the shadow cone of one surface atom (A) just intersects its nearest surface neighbor as shown in Fig. 10a, $\underline{C}$ is equal to the distance between these two surface atoms. If this quantity is known by e.g. Low Energy Electron Diffraction (LEED), then it is possible to define a relation between $\underline{\mathrm{R}}$ and $\underline{r}$ for the specified ion-atom interaction. By varying the angle of incidence of the primary beam, a second critical angle $\psi_{\mathrm{c}}$ (Fig. 10b) may be found for which the second layer (B) atoms lie on the shadow cone surface. Since $C^{\prime}, R^{\prime}$ and $r^{\prime}$ form a right triangle and the relation ship between $R^{\prime}$ and $r^{\prime}$ is now known, it is possible to determine the interatomic distance and bond angles between atoms in the first few atomic layers [7,38,53-61].

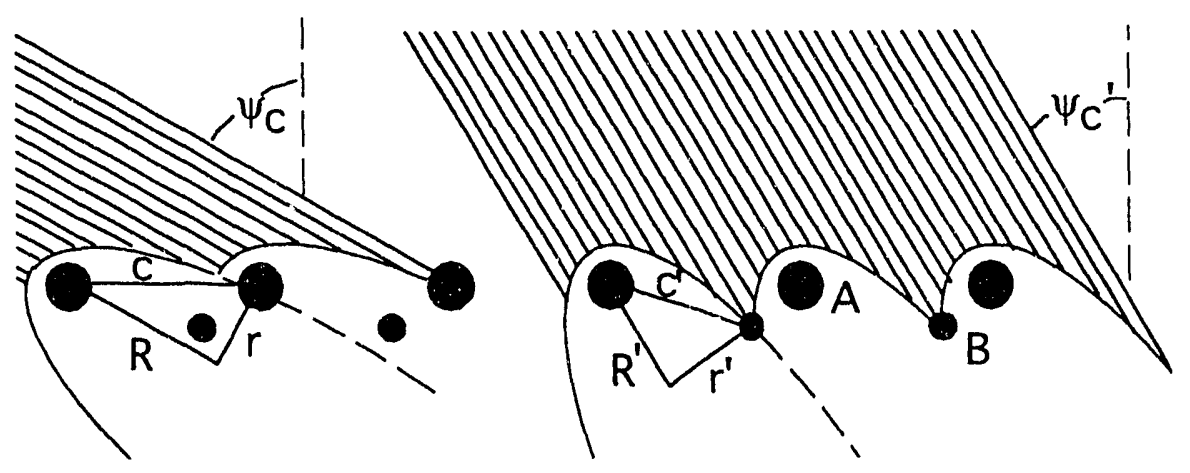

Fig. 10. Determination of the interatomic bond distance and angle from shadow cone shape analysis.

In practice, the interpretation of surface structure from shadow cone data as depicted in Fig. 10 is not always straightforward. The critical angle is measured as the angle at which there is an enhanced signal for the shadowed atom species. However, as shown in Fig. 11, the angle of incidence at which signal enhancement is observed depends on the placement of the detector. In Fig. $11 \mathrm{a}$, the scattering angle is $\theta=180^{\circ}$, placing the ion source and detector along the same axis. Enhancement of the atom B scattering signal occurs for an angle of incidence $\psi_{\mathrm{C}}$, corresponding to the condition that the shadow cone of atom $\mathrm{A}$ just intersects atom B. For scattering angle $\theta^{\prime}<180^{\circ}$ (Fig. $11 \mathrm{~b}$ ), the enhancement of the atom $B$ signal occurs at an angle of incidence $\psi \mathrm{c}^{\prime}$ which does not correspond to the condition that atom $\mathrm{B}$ lies on the shadow cone of atom A. Therefore the quantitative analysis described in the preceding paragraph is only valid if the detector is placed as close as possible to the axis of the incident ion beam. This is usually done by using a relatively small detector placed close to the axis of the ion source. Ion scattering spectroscopy performed in the geometry with $\theta$ close to $180^{\circ}$ is sometimes referred to as "Impact Collision ISS" (ICISS) [58]. The term "CAICISS" [40] refers to the experimental configuration in which a coaxial detector is used to ensure that the scattering angle is as close to $180^{\circ}$ as possible. "ALICISS" [28] refers to the use of an alkali ion beam in conjunction with the ICISS geometry, and "NICISS" [31] refers to the detection of backscattered neutrals using ToF detection in the ICISS geometry. 


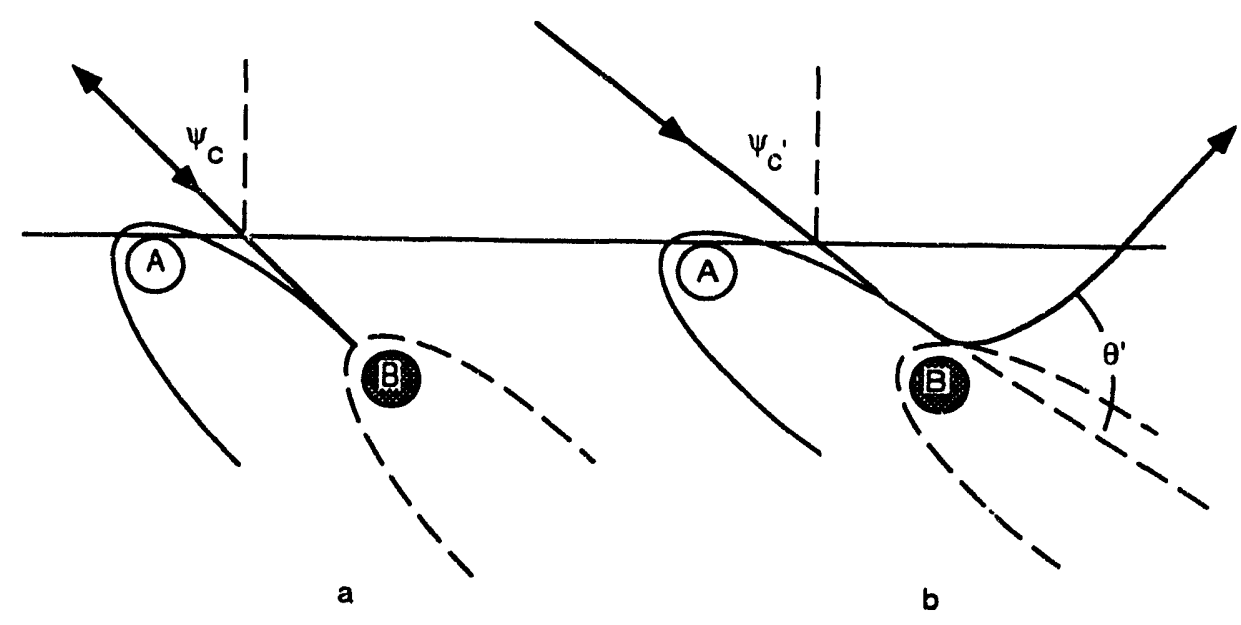

Fig. 11, A comparison of the shadow cones for two scattering geometries: $\theta=180^{\circ}$ (a), and $\theta^{\prime}<180^{\circ}$ (b).

An example of the difference between ICISS with detection of backscattered inert gas ions and alkali ions or neutral inert gas atoms is shown in Fig. 12 for the Pt(111) surface. The collision geometries, labelled 1,2 and 2' are shown in the upper part of Figure 12 for three different angles of incidence. Gecmetry 1 refers to oblique incidence with consequent scattering by a first layer atom. Geometry 2 refers to a steeper angle of incidence with scattering by an exposed second layer atom without any interaction with first layer atoms. Geometry $2^{\prime}$ refers to an even steeper angle of incidence with consequent scattering from a second layer atom followed by a grazing collision with a first layer atom along the exit trajectory. The corresponding spectra for inert gas and alkali metal ions are shown in the lower part of Figure 12. The inert gas ion signal is dominated by charge exchange events arising from long range electronic interactions rather than the nuclear collision events which reflect the crystal structure. For the alkali ions however, the three types of collisions are clearly reflected in the polar angle scan of the backscattering intensity.

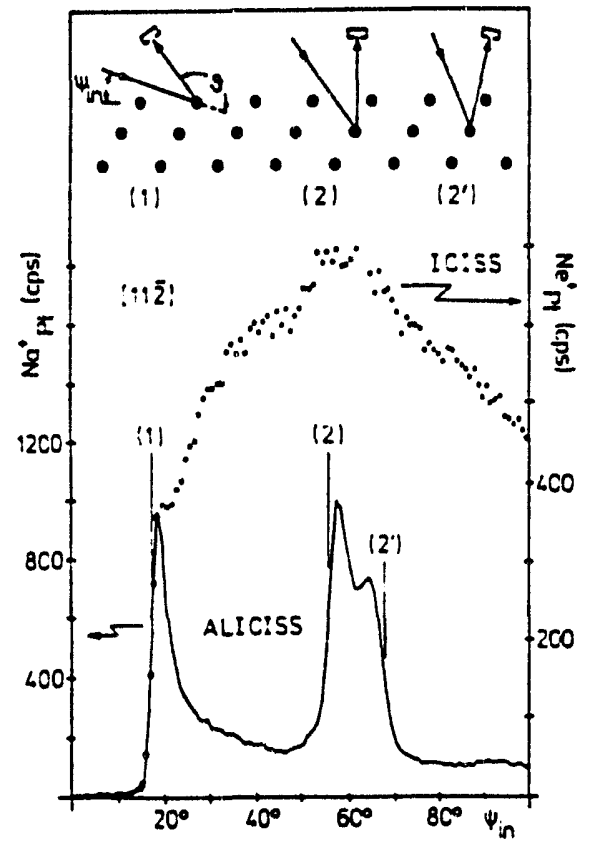

Fig. 12. $\Psi_{\text {in }}$ patterns of $\mathrm{Na}^{+}$(solid line) and $\mathrm{Ne}^{+}$ions (dots) backscattered from $\mathrm{Pt}$ atoms as measured at constant energy with an electrostatic energy analyzer. Scattering angle $\theta=45^{\circ}$, incident plane in the [112] azimuth of Pt (111) [after H. Niehus and G. Comsa (1986)] 
ICISS has been used [55,58] to quantify the structure of the TiC (001) surface. Qualitatively, the structure is similar to that of Fig. 11, with the carbon atoms residing slightly below the surface. The shape of the shadow cone for $1 \mathrm{keV} \mathrm{He}+$ incident on $\mathrm{Ti}$ has been determined by measuring the $\mathrm{Ti}$ critical angles for the (111) and (001) surfaces of $\mathrm{TiC}$, (Fig. 13), fitting the measured $\mathrm{Ti}$ critical angles and an assumed Thomas-Fermi-Molière potential [62]. The resulting shadow cone is shown in Fig. 14. For $1 \mathrm{keV} \mathrm{He}+$ incident along the $<100>$ azimuth of the $\mathrm{TiC}(001)$ surface, the critical angle for the carbon signal occurs at $\alpha_{c}=24^{\circ}$. From this it may be determined [58] that the $C$ atoms are located $0.87 \pm 0.08 \AA$ below the surface and $1.97 \pm 0.05 \AA$ from the nearest $\mathrm{Ti}$ atom, at an angle $26 \pm 1^{\circ}$ relative to the plane of the surface [55].

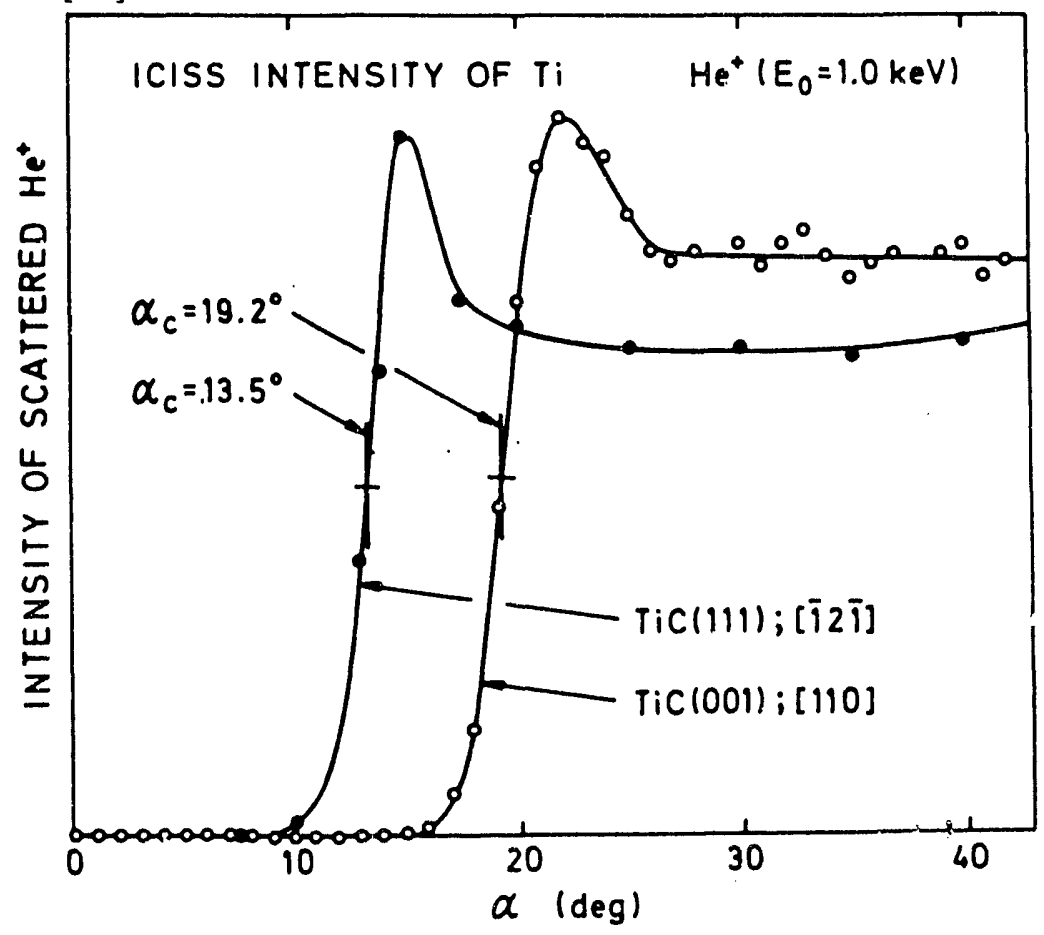

Fig. 13. Angular resolved intensity dependence of the Ti signal from TiC (111)-1x1 surface. (After Aono 1984)

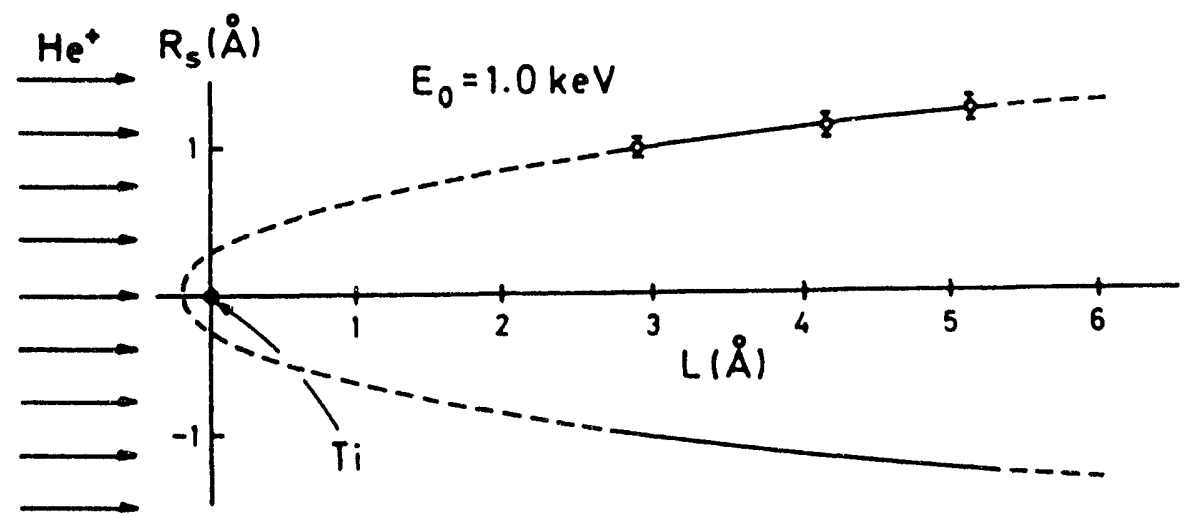

Fig. 14 Shape of the shadow cone of Ti for $1 \mathrm{keV} \mathrm{He}^{+}$incident ions. (After Aono, 1984) 
2.2.5 Surface Defects. If there are surface defects as indicated in Fig. 15 in which one of the $A$ atoms is missing, then some $B$ atoms will be visible for angles of incidence in which the B atoms would all be in the shadow cone of the $A$ atoms if there were no lattice defects. Consequently, there would be a bump in a plot of the peak intensity corresponding to element B as a function of angle of incidence. An example is shown in Fig. 16 for TiC (001). In this case, a carbon vacancy permits the detection of $\mathrm{Ti}$ atoms below the critical angle, giving rise to the structure indicated by the shaded area.

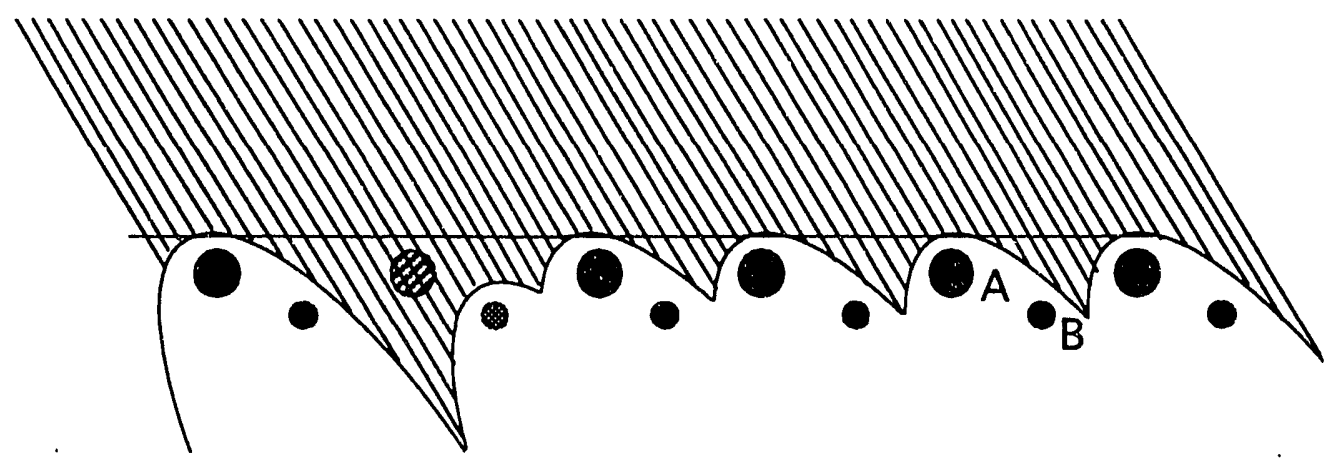

Fig. 15. All the B atoms except for one (grey) are inside the shadow cone of atom A. A missing surface atom $A$ (hatched) allows a B atom to be exposed to the incident primary ion flux.

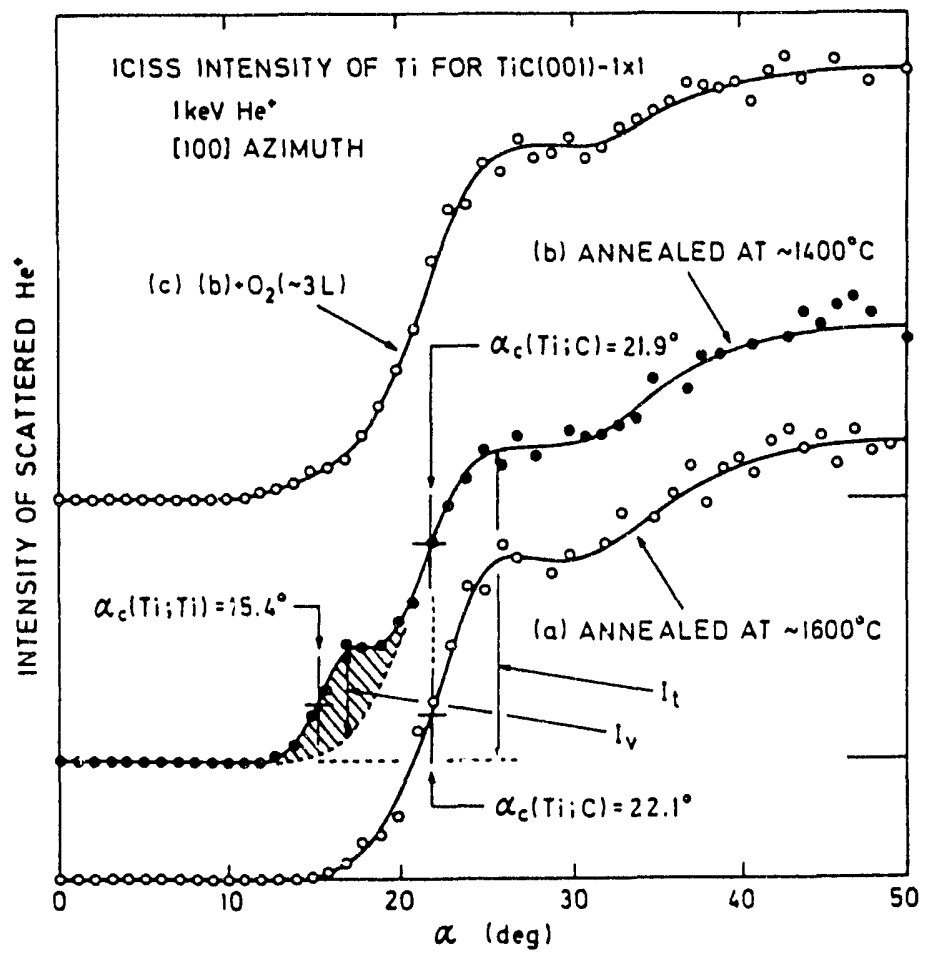

Fig. 16. Angular variation of the Ti ISS signal for a TiC surface (a) annealed, (b) with missing $\mathrm{C}$ atom defects, and (c) after oxygen adsorption which results in filling of the defect sites by oxygenatoms. (After Aono 1984). 
2.2.6 Interface Analysis. King et. al. [63] have applied CAICISS to interface analysis during thin film growth. With careful interpretation of the angular intensity profiles, they are able to probe 10 monolayers or more across an interface to determine the registry of film and substrate atoms. Deposition of a $\mathrm{CaF}_{2}$ epitaxial film on a $\mathrm{Si}$ (111) substrate was monitored using a $2 \mathrm{keV} \mathrm{He}+$ probe beam, with a typical dose of $3 \times 10^{10}$ ions/spectrum. Angular scans of the $\mathrm{Ca}$ and $\mathrm{Si}$ signals for a $1 \mathrm{ML}$ film of $\mathrm{CaF}_{2}$ on a $\mathrm{Si}(111)$ substrate show similar peaks, but with the Si peaks reversed about the $90^{\circ}$ angle of incidence, showing that the $\mathrm{CaF}_{2}$ layer and $\mathrm{Si}$ have identical structures, but with the $\mathrm{CaF}_{2}$ epitaxial layer rotated $180^{\circ}$ with respect to the substrate. The structure for the $\mathrm{CaF}_{2}$ layer is however, different from that of bulk $\mathrm{CaF}_{2}$. For thicker films, $(\sim 500 \AA)$ grown at a temperature of $700^{\circ} \mathrm{C}$ and annealed to $750{ }^{\circ} \mathrm{C}$ for 8 minutes, the $\mathrm{Ca}$ angular profile shows new structure which is consistent with the assumption that it corresponds to $\mathrm{CaSi}_{2}$ (Fig. 17). Modeling the CAICISS peak intensities by the computer code TRIM, it is determined that the $\mathrm{CaSi}_{2}$ layer exists at a depth of at least 13-40 $\AA$ below the surface. Therefore it is concluded that although the initial growth of $\mathrm{CaF}_{2}$ on $\mathrm{Si}$ (111) is epitaxial, an intermediate $\mathrm{CaSi}_{2}$ layer is formed prior to establishment of the bulk $\mathrm{CaF}_{2}$ structure.

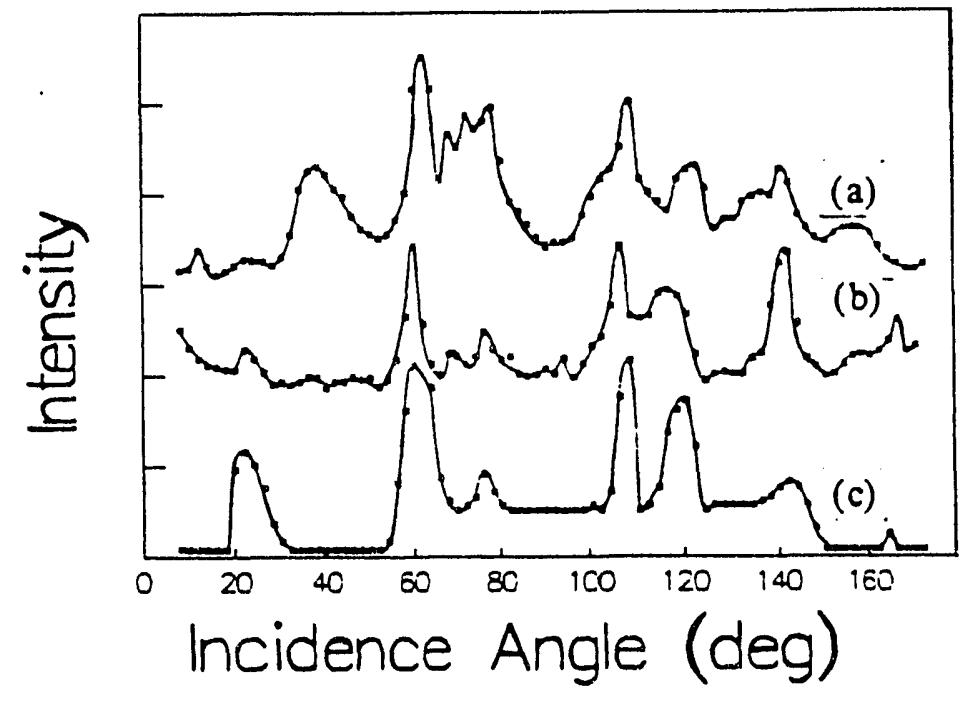

Fig. 17 CAICISS yield from the Ca surface peak as a function of the angle of incidence of $2 \mathrm{keV} \mathrm{He}+$ with respect to the sample surface for (a) an epitaxial $\mathrm{CaF}_{2}$ film deposited on $\mathrm{Si}(111)$ at $700^{\circ} \mathrm{C}$. (b) the above film after annealing at $750^{\circ} \mathrm{C}$ for 8 minutes. (c) a numerical simulation of ion scattering from $\mathrm{Ca}$ terminated $\mathrm{CaSi}_{2}$. (After King et. al., 1990)

\subsection{DIRECT RECOIL SPECTROSCOPY (DRS)}

By placing a detector in the forward scattering direction, surface atoms ejected by direct recoil sputtering are seen in addition to the scattered primary beam. Detection of these recoilsputtered atoms constitutes the basis of the closely related Direct Recoil Spectroscopy (DRS) analysis technique. ISS provides no signal for ions lighter than the probe beam, but DRS is one of the few surface analytical techniques which is sensitive to helium and hydrogen, as shown in Fig. 18, and is able to distinguish between $\mathrm{H}$ and $\mathrm{D}$ at levels down to about $1 \%$ [64]. The data in this Figure represents the surface composition of a $\mathrm{Cu}-\mathrm{Li}$ alloy which forms a very thin (1-2 monolayer) lithium surface layer by Gibbsian segregation, as shown in Fig. 18a. In Fig. 18b, a fraction of a monolayer of $\mathrm{Cu}$ is evaporated onto the surface, resulting in a decrease in the $\mathrm{Li}$ peak signal and an increase in the $\mathrm{Cu}$ signal. With the adsorption of $10 \mathrm{~L}$ of $\mathrm{CH}_{3} \mathrm{OD}$, the $\mathrm{H}, \mathrm{D}$ and $\mathrm{C}$ levels increase significantly, while the $\mathrm{Cu}$ signal decreases slightly and the Li signal decreases significantly, indicating a preferential adsorption on Li-terminated surfaces. Previous studies of this material $[65,66]$ by AES have failed to determine whether 
oxygen preferentially adsorbed on the Li-covered portion of the surface, or whether it was distributed uniformly over both the $\mathrm{Li}$ and $\mathrm{Cu}$-covered portions of the surface.

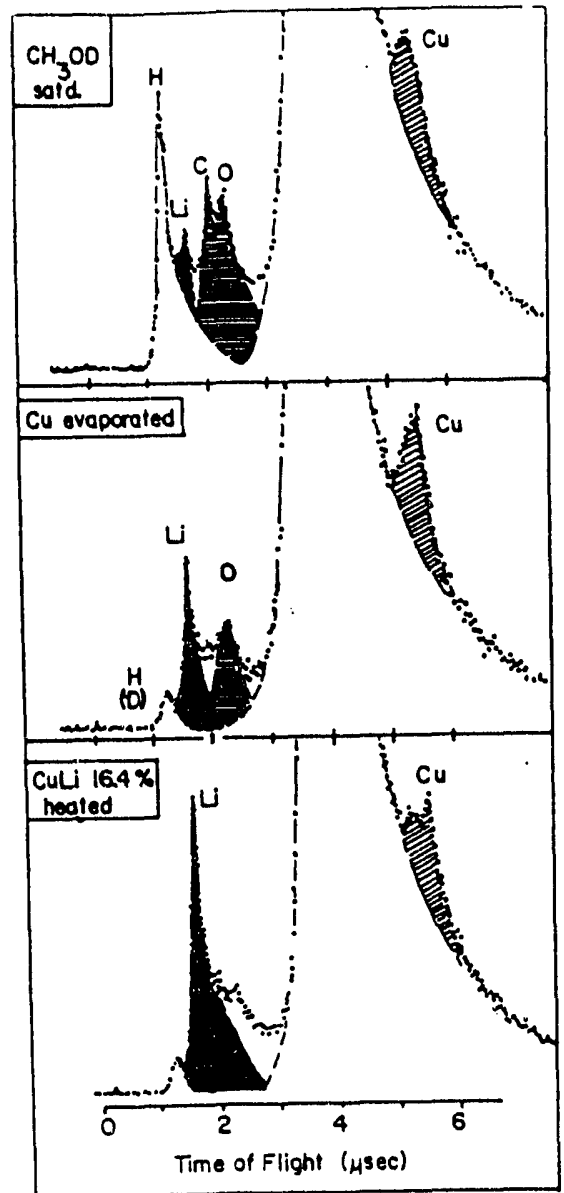

Fig. 18 Direct Recoil Spectra of a lithium-covered Cu-Li alloy surface. (a) segregated lithium overlayer (b) deposition of $\angle 1 \mathrm{ML}$ of $\mathrm{Cu}$ on the surface of Fig. $18 \mathrm{a}$. (c) $10 \mathrm{ML}$ of $\mathrm{CH}_{3} \mathrm{OD}$ deposited onto the surface of Fig. 18a. (After Schmidt et. al. )

Because of the long source-detector distances associated with the ToF detection scheme, the analysis method does not interfere with the equipment required for the thin film deposition process. It has been demonstrated $[40,67]$ that Time-of-Flight Ion Scattering Spectroscopy (ToF-ISS) can be used as an in situ monitor of thin film surface composition for low-pressure deposition processes such as MBE. However, the scattering mean free path of low keV ions is much longer than that of sub-keV electrons, and DRS and ISS are also much more tolerant of high background pressures than most other surface analytical methods, although they are simultaneously much more surface-specific than other surfaceanalytical methods. It is possible to provide differential pumping of the incoming and outgoing ion beam paths to extend the pressure limits of the ToF ion beam techniques even further, as shown in Fig. 19. This capability has been demonstrated for DRS of diamond-like films during growth by Low Pressure Chemical Vapor Deposition (LPCVD) at pressures up to 1 Torr, and hydrogen adsorption has been measured on (100) diamond at ambient pressures up to 330 mTorr with very little loss of resolution [64] as shown in Fig. 20. This represents a 6-8 order of magnitude increase in the permissible operating pressure compared with other surface analytical methods. At higher beam energies, it has been demonstrated that 
accelerator-based ion beam analysis is even possible at atmospheric pressure [68] although surface specificity is lost.

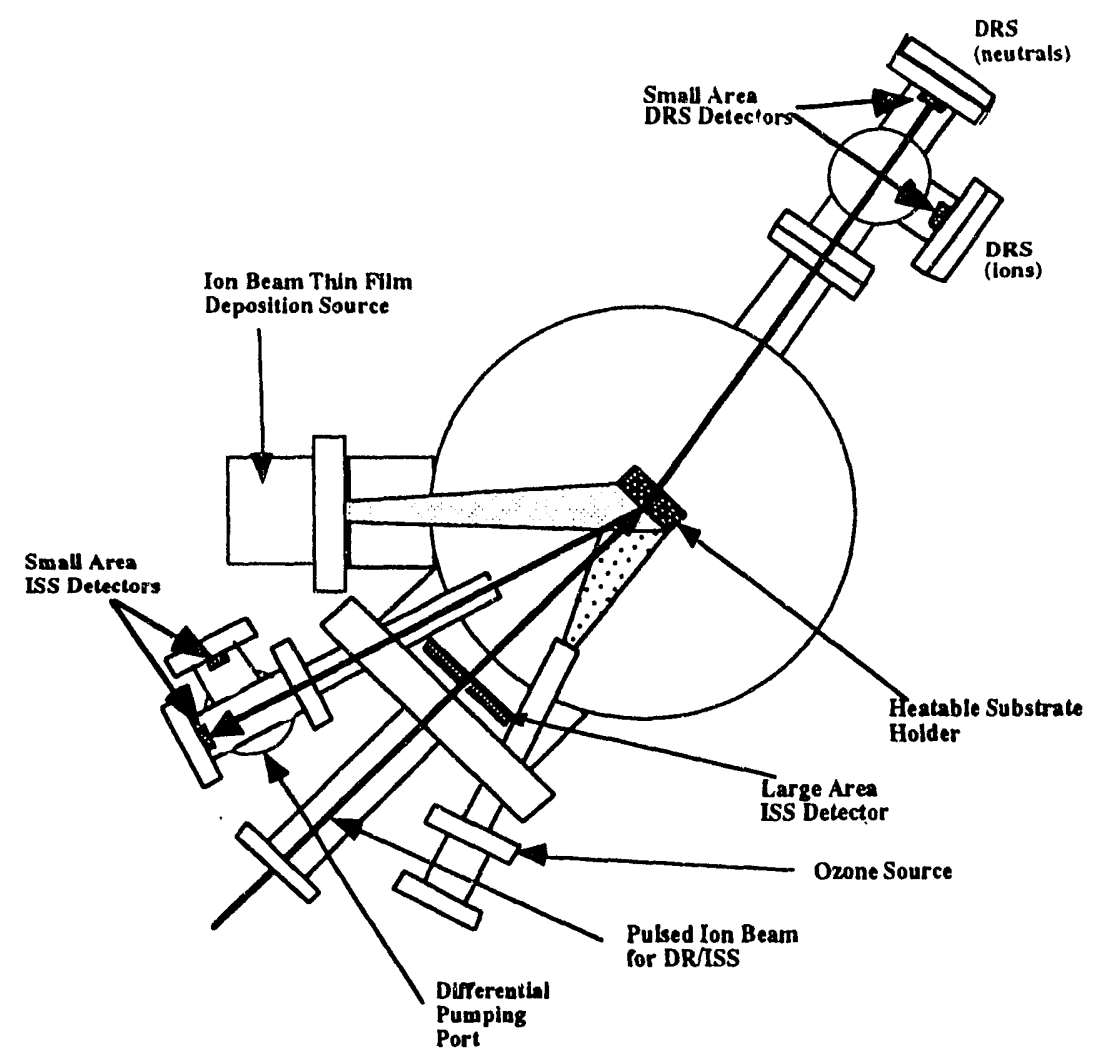

Fig. 19 Differentially pumped pulsed ion beam analysis/ion beam thin film deposition chamber, showing small and large detectors for ISS and DRS.

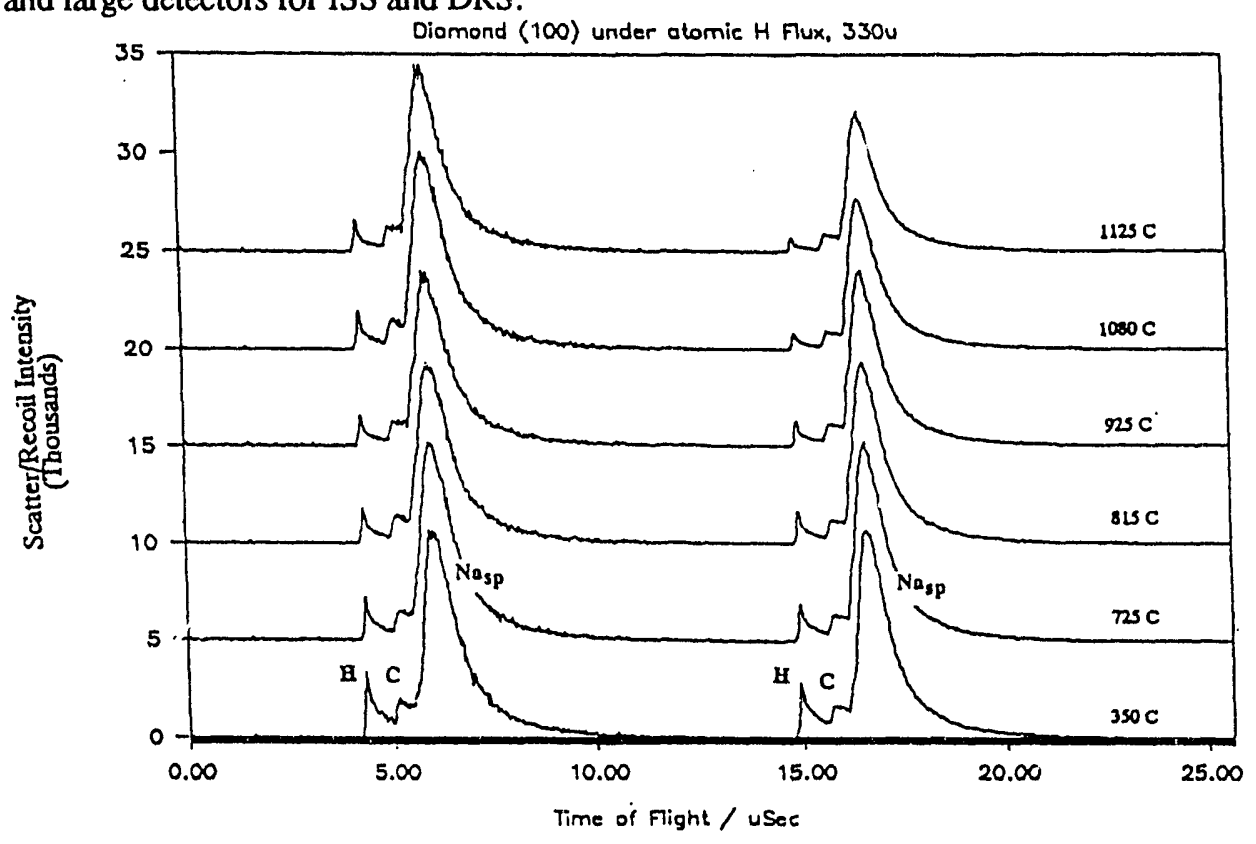

Fig. 20. Direct Recoil spectrum of a diamond (100) film exposed to a flux of atomic hydrogen in an ambient pressure of $330 \mu$ Torr. Data was taken using a $6 \mathrm{keV} \mathrm{Na}^{+}$bearn. (After Schmidt et. al. 1990) 


\subsection{MASS SPECTROSCOPY OF RECOILED IONS (MSRI)}

A technique related to ISS and DRS, Mass Spectroscopy of Recoiled Ions (MSRI), has been developed by Ionwerks, Inc. As shown in Fig. 19, the neutral direct recoils impinge on an electron multiplier which is in line of sight with the target. The output of this detector constitutes the DRS signal. The recoiled ions however, may instead be deflected into a Poschenrieder-type mass analyzer. The instrumentation is very similar to that of ToF SIMS except that the positioning of the analyzer preferentially detects direct recoil ions. The DR ions have much higher kinetic energy than the SIMS ions and are therefore able to penetrate a region of much higher ambient pressure without significant scattering. Consequently, the pressure limit of MSRI is expected to be similar to that of ISS and DRS. The ultimate sensitivity and mass resolution appear to be similar to that of ToF-SIMS except that as a result of the higher kinetic energy associated with the DR process, there is less neutralization of the ejected ions in the near-surface region, and consequently a reduction in the undesirable matrix effect which makes SIMS very difficult to quantify. For elements such as boron, in which the SIMS secondary ion fraction is very low, MSRI may be more sensitive. A MSRI spectrum of a BN film, obtained in 20 seconds, is shown in Fig. 21. The demonstrated resolution is significantly better than most quadrupole-based SIMS instruments. A recent design modification has improved the MSRI signal to noise ratio and resolution even further, yielding a dynamic range of $10^{9}$ (sensitivity $<1 \mathrm{ppb}$ ) and mass resolution of 400 at mass 238. The time necessary to acquire a spectrum comparable to Fig. 21 is now well under one second. It is anticipated that MSRI will be extremely valuable for real-time quantitation of majority elements, dopants, impurities, and film-substrate interdiffusion during thin film deposition, perhaps achieving new detection limits for trace elements where SIMS has limited sensitivity (e.g., $\mathrm{N}$ in GaAs). Because of the absence of the SIMS matrix effect, the results, can be made quantitative to within $\sim 5 \%$, compared to a typical value of $\pm 50 \%$ for SIMS .

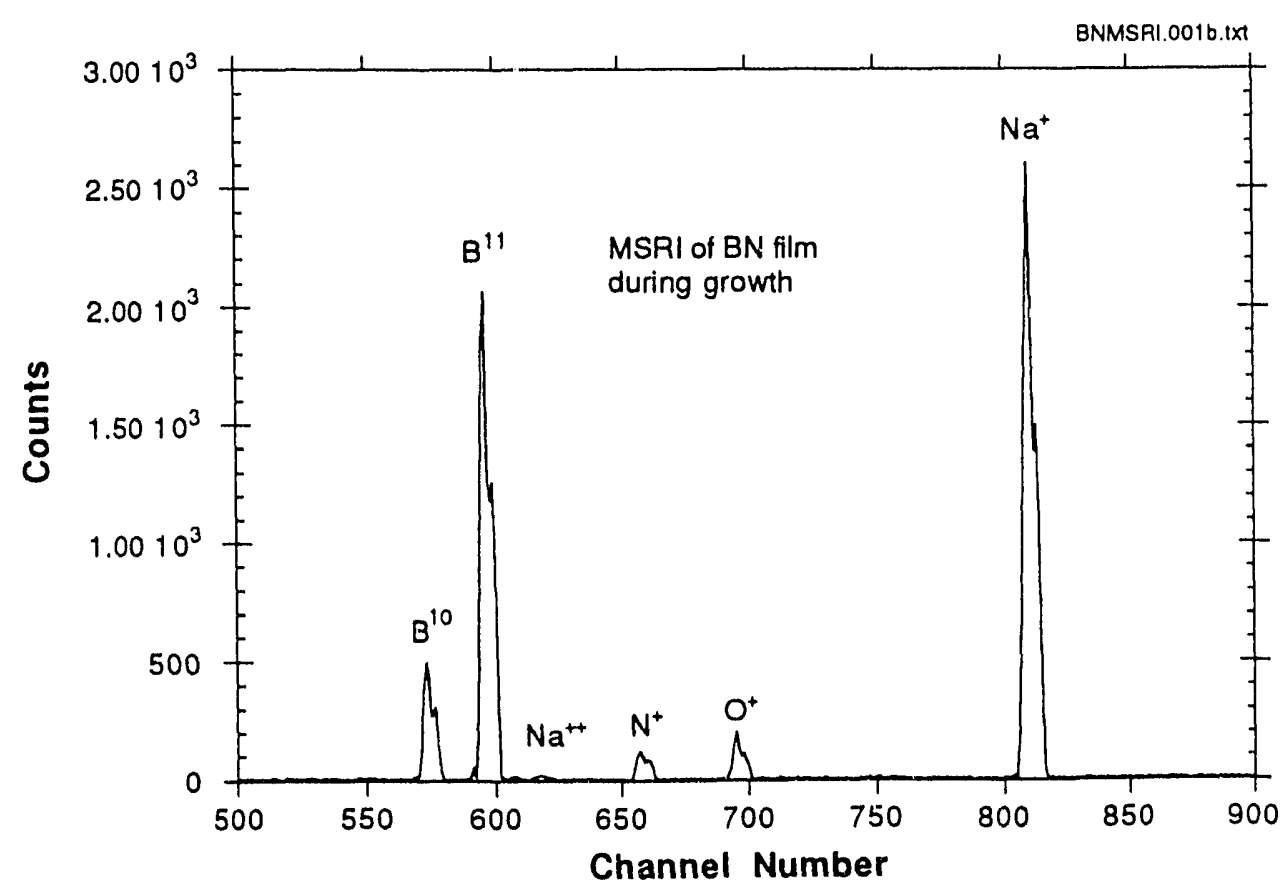

Fig. 21 MSRI spectrum of a BN film during growth at $10^{-4}$ Torr. 


\subsection{Instrumentation}

A unique Time-of-Flight Direct Recoil and Ion Scattering Spectrometer (ToF-DR/ISS) system is currently under development at Argonne National Laboratory. This system (shown in Fig. 19), has been used to demonstrate extremely low dose surface analysis, very high data acquisition rate, and considerably greater flexibility of operation than previous designs [69]. A thin film multi-target ion beam growth chamber [70] has been added as an appendage to the analysis chamber, permitting real-time monitoring of the growth of multicomponent metal and metal oxide thin films and layered structures.

3.0.1 Pulse Formation. The beam line is depicted in Fig. 22. It consists of a telefocus ion source injecting a 5-12 keV mass analyzed ion beam into a transfer section containing two deflection regions separated by a drift space. A dc beam current of 1-2 $\mu \mathrm{A}$ may be directed through the beam line onto the sample at $10 \mathrm{keV}$. The apertures A1-A4 may be adjusted in situ, selecting from 6 sizes ranging from $250 \mu \mathrm{m}$ to $4 \mathrm{~mm}$ in diameter. The current reaching the sample is proportional to the area of the selected aperture size as shown in Fig. 23, indicating that the beam is highly paraxial. For pulsed operation, the deflection voltages are added to small dc offsets which are adjusted to provide maximum beam current when the apertures are slightly out of alignment in order to eliminate energetic charge-exchange neutrals from the beam. The voltage pulses which are applied to the deflection plates are of fixed width, with very short rise time. The deflection plates run nearly the full distance between the apertures, thereby providing high deflection sensitivity. Fringe field effects are also eliminated since ions which are not very close to the axis as they leave the deflection field will not enter the aperture at the end of the deflection region.

Initially, no voltage is applied to the A stage and the beam therefore passes through apertures A1-A3. However, a deflection voltage of 100 volts is applied to the B section of the beam line, preventing transmission through aperture A4. A deflection voltage is then applied to section A, cutting of the tail end of the ion column as shown in Fig. 22. The tail end then travels through the field free region between the two deflection sections. At a predetermined time shortly before the end of the ion column enters the B section, the B deflection voltage is removed, permitting a short burst of ions to pass through aperture A4 to the sample. The time between the leading edge of the first pulse and the falling edge of the second pulse determines the temporal length of the ion beam pulse. The data acquisition software determines the timing to provide the ion beam pulse length specified by the operator. The pulse length can be adjusted from $\sim 10 \mathrm{~ns}$ to $1 \mu \mathrm{s}$. It is therefore possible to adjust the pulse length to suit the resolution and count rate needs of the measurement.

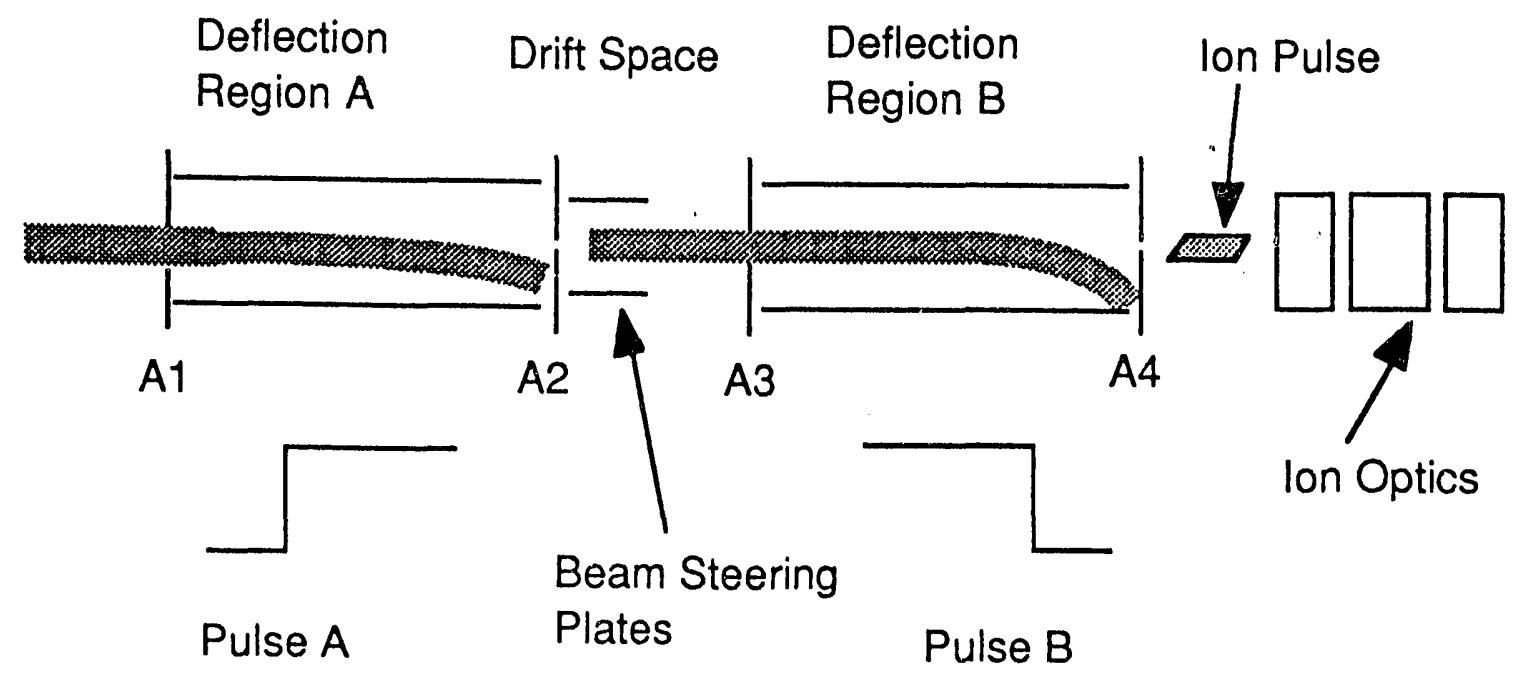

Fig. 22 Schematic diagram of the Dual Pulsed Bean Line with microfocus capability. 


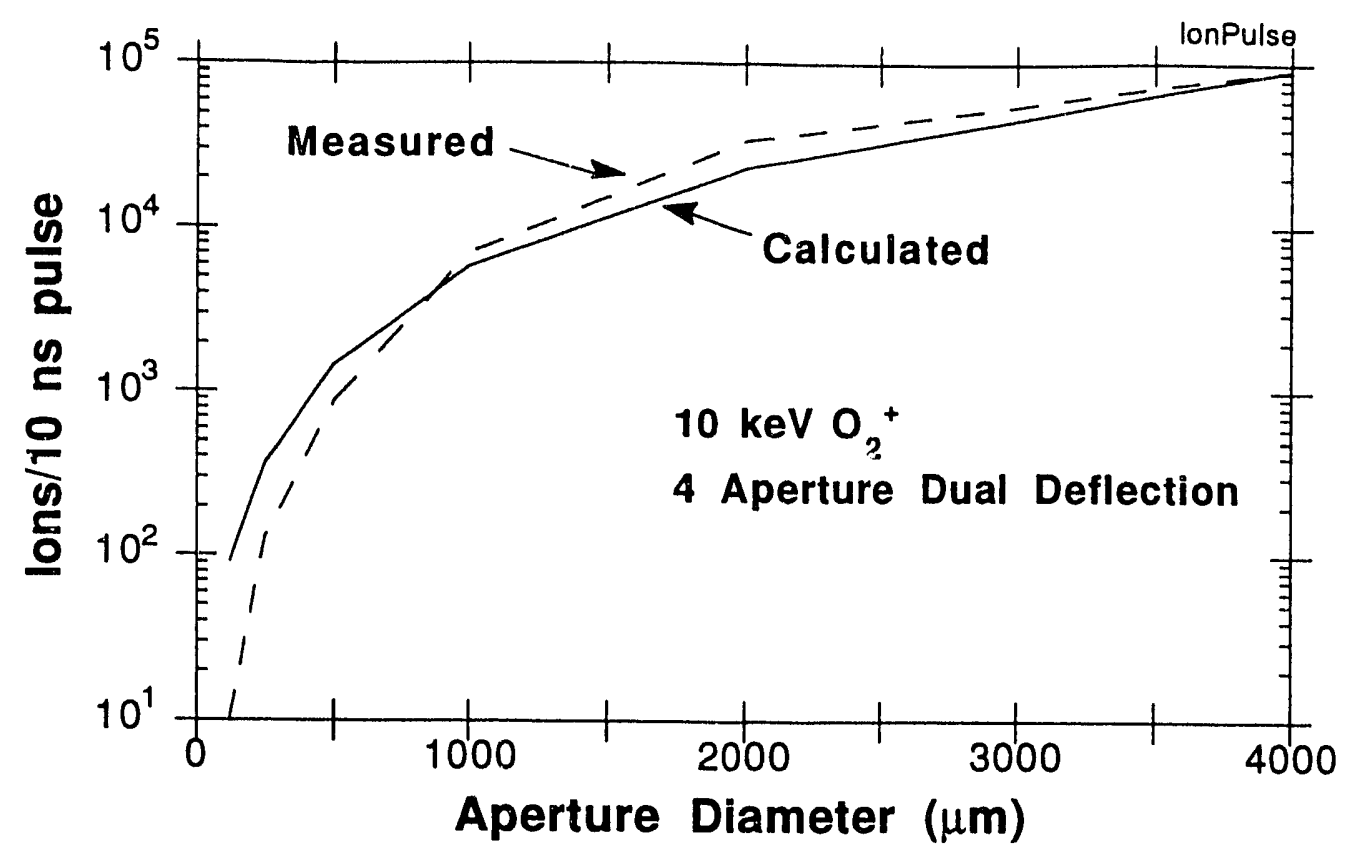

Fig. 23. Plot of the calculated and measured beam current incident on a sample located $30 \mathrm{~cm}$ from the exit aperture of the beam line shown in Fig. 22 as a function of aperture size.

A ToF-ISS spectrum obtained for $10 \mathrm{keV} \mathrm{Ne}+$ incident on stainless steel 304 is shown in Fig. 24. In this figure, both scattered ions and neutrals were detected, using the ToF-ISS

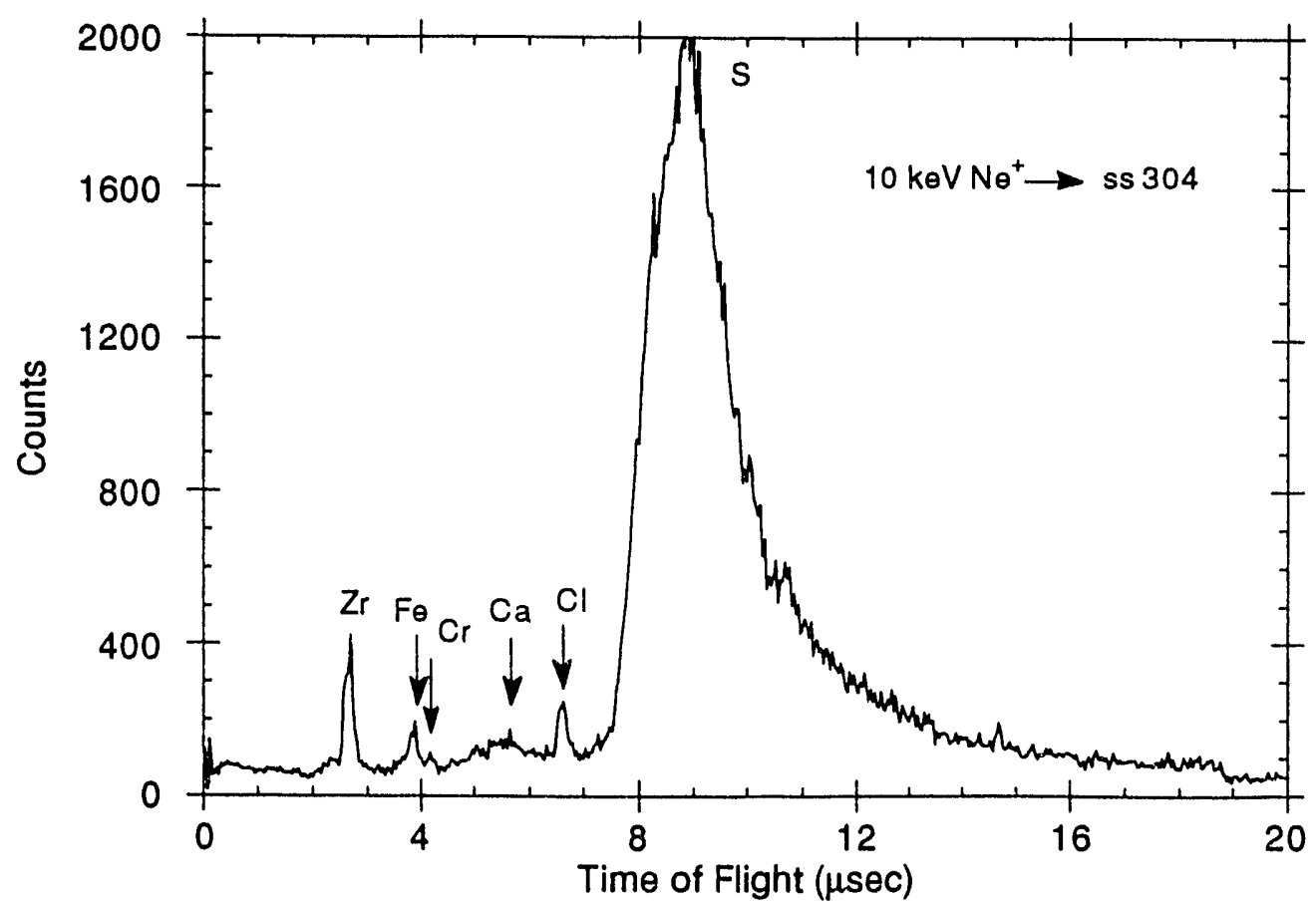

Fig. 24. Time-of-flight ion scattering spectrum of 304 stainless steel, caken with a $10 \mathrm{keV} \mathrm{Ne}{ }^{+}$ion beam using a pulse length of $100 \mathrm{~ns}$. 
analyzer under development at Argonne National Laboratory. The largest peaks are seen for segregated $\mathrm{S}$ and $\mathrm{Cl}$, which are presumably present as surface contaminants. $\mathrm{Fe}$ and $\mathrm{Cr}$ were detected, but at much lower intensity. The peak at $2.68 \mu \mathrm{s}$, identified as $\mathrm{Zr}$, is the only element which is neither a constituent nor a common surface impurity in stainless steel. Auger spectroscopy obtains its signal from a somewhat greater depth than ISS and may provide different elemental abundances than ISS. An Auger spectrum of this sample reveals relatively small amounts of $\mathrm{S}$ and $\mathrm{Cl}$, large $\mathrm{O}$ and $\mathrm{Fe}$ peaks, a small amount of $\mathrm{Ni}$ and no $\mathrm{Cr}$ or $\mathrm{Zr}$.

A short ion beam pulse provides good mass resolution and exhibits a distinctly asymmetric peak, as shown in Fig. 25, for detection of both ion and neutral Ar scattered into a small area detector from a sample of 304 stainless steel. Auger analysis, which has a sampling depth of 10-15 $\AA$, shows a surface consisting largely of $\mathrm{Fe}$, with almost no $\mathrm{Cr}$ visible. The large Cr peak in Fig. 25 results from the fact that ISS is primarily sensitive to the composition of the first atomic layer, which is strongly enriched in $\mathrm{Cr}$. The long flight time tail of the Cr peak results from scattering of the primary beam by atoms in the second, third, and fourth atomic layers. A similar asymmetry has been observed in DRS data, and the shape has been calculated by Eckstein [71]. In principle, the shape of the long flight time tails can be deconvoluted to provide information on the depth distribution of each atomic species in the first few atomic layers.

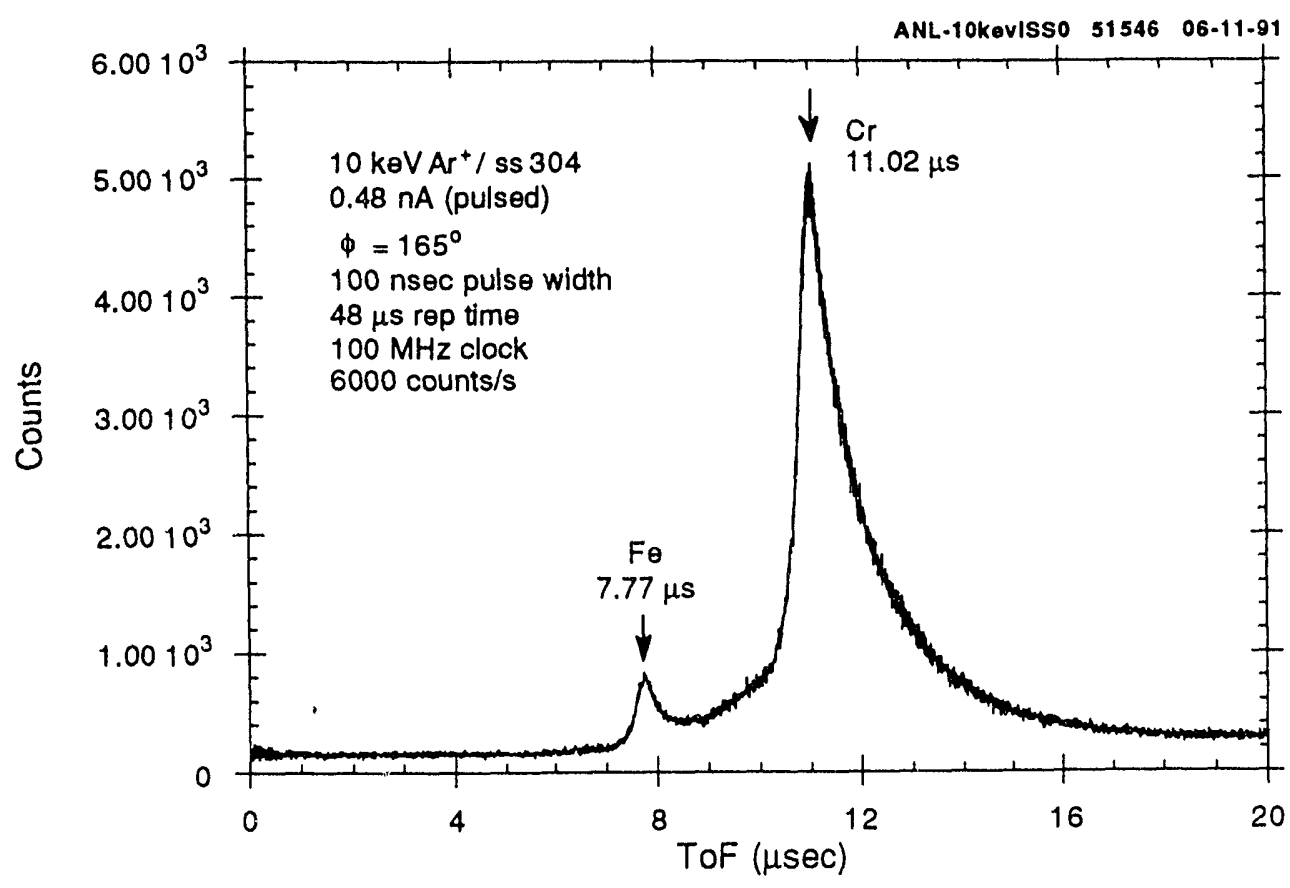

Fig. 25 ToF-ISS spectrum of stainless steel taken with a $1-\mathrm{cm}$ diameter detector.

A long ion beam pulse results in decreased mass resolution, as shown in Fig. 26, but provides significantly increased count rate. The total ion dose used for the spectra of Figs. 25 and 26 was $\sim 10^{12}$ ions. However, the mass resolution of Fig. 26 is more than adequate for elemental identification and it is possible to obtain useful data with even lower ion doses $\left(\sim 10^{10}\right.$ ions), as shown in Fig. 27. 


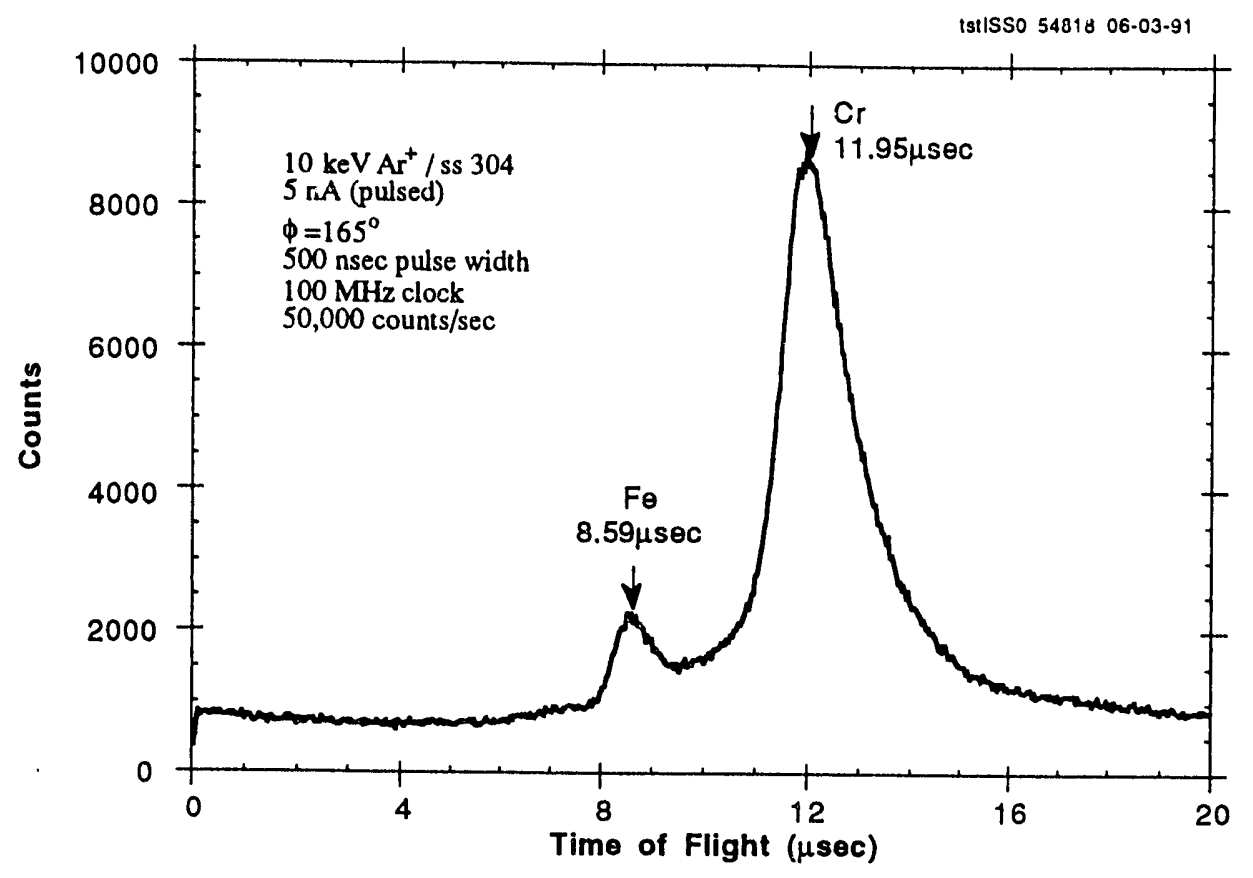

Fig. 26 ToF-ISS spectrum of stainless steel taken with a $1-\mathrm{cm}$ diameter detector in the ANL DR/ISS system using a 500 nsec pulse width.

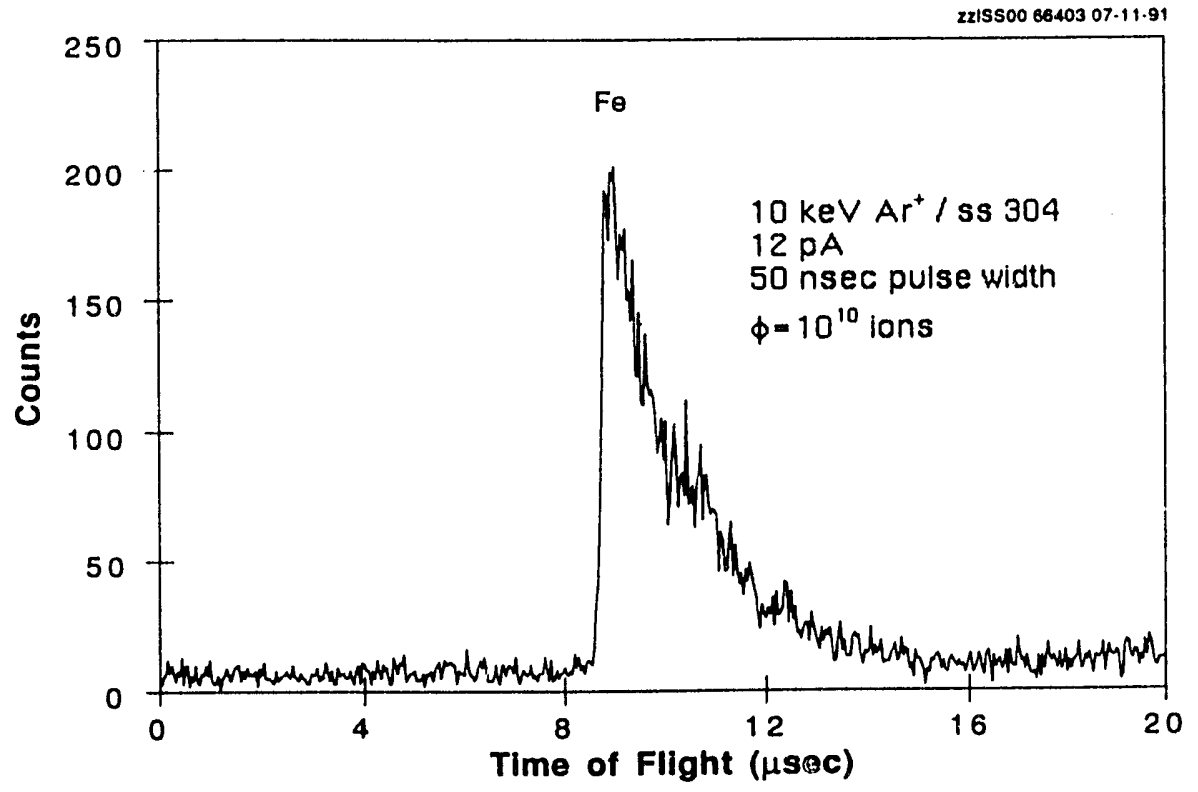

Fig. 27 ToF-ISS spectrum of stainless steel taken with a $1-\mathrm{cm}$ diameter detector in the ANL DR/ISS system using a beam dose of $10^{10}$ ions. 
The spectra shown in Figs. 25-27 were taken using a small $(1 \mathrm{~cm}$ diameter) spiraltron detector. As described below, the installation of a large area channel plate detector will reduce the required dose an additional two orders of magnitude. At that point, the ion dose will be competitive with the much more complex and expensive laser resonance ionization technique, which currently holds the record as the most sensitive surface analytical method known.

\subsection{BEAM CHARACTERISTICS}

The transmitted ion pulse forms a footprint on the sample surface consisting of a stationary "hotspot" along with a much less intense "corona" arising from the tail of the chopped ion beam, which moves as the beam is pulsed. Details of the pulse characteristics will be presented elsewhere [72]. For long ion beam pulses and short rise time deflection voltages, the current contained in the corona becomes negligible in comparison with the stationary hotspot. The hotspot can be focused and rastered across the surface, thereby functioning as a microprobe. Because of the high count rate associated with the use of long ion beam pulses and the large area detector (described below), reasonably short data acquisition times of scanned images are possible, limited primarily by the speed with which the computer is able to read the data from the histogram memory and assemble the images. Preliminary results indicate that good imaging signal/noise should be obtained with a dose of $10^{7}-10^{8}$ ions per pixel. We define the term "static" ion beam dose as a dose which is small enough to cause negligible damage, corresponding to a maximum of approximately $1 \%$ of the atoms in the surface area exposed to the beam, or typically about $10^{13}$ ions $/ \mathrm{cm}^{2}$. The smallest spot which may be resolved by the Argonne DR/ISS system using the large area detector, while not exceeding this dose is $\sim 5 \mu \mathrm{m}$ in diameter. Using a light probe ion such as He which results in both a reduced damage level and a higher fraction of backscattered primary ions, the diameter of the minimum area which may be resolved while keeping the damage below the $1 \%$ level is approximately $1 \mu \mathrm{m}$. By comparison, the corresponding beam diameter for the 127 degree ESA used by McKinney and Frankenthal [4] is $10 \mathrm{~cm}$.

\subsection{DETECTORS}

The system has five detectors, of which four are differentially pumped, permitting analysis of samples which are at ambient pressures approaching one Torr. The differentially pumped detectors are spiraltron electron multipliers with a $1 \mathrm{~cm}$. diameter collection cone, located approximately $50 \mathrm{~cm}$ from the sample. They are geometrically arranged so that there are two detectors, one line of sight and the other off-axis, permitting independent detection of ions and neutrals in both the forward and back-scattering directions. The fifth detector is a 4 $\mathrm{cm}$. diameter channel plate with a segmented anode collector consisting of 8 concentric rings, each connected to a separate preamplifier and discriminator, located $25 \mathrm{~cm}$ from the sample. Each segment corresponds to scattering into a well-defined polar angle, and therefore a peak in the ToF spectrum corresponds to a well-defined surface species with mass $M_{2}$. The spectra collected by each segment, are stored separately in a CAMAC histogram memory. The timeof-flight axis is converted to an $\mathrm{M}_{2}$ scale by the data acquisition software and the spectra from the various detector segments are then added to produce a composite spectrum which has the same mass resolution as that of the differentially-pumped small area detectors but $64 \mathrm{x}$ higher count rate (or equivalently, $64 x$ lower beam dose for the same signal/noise ratio). The high data acquisition rate obtainable with this detector, coupled with the high count rate provided by the use of relatively long ion pulses and the stable beam position resulting from the dual beam chopping scheme will permit the development of a scanning ISS microprobe, and is expected to reduce the time required for a complete polar-azimuthal angular scan from approximately 10 hours to 10 minutes. 


\subsection{DATA ACQUISITION}

The ability to use long beam pulses, along with a high heam current and the extremely high signal collection efficiency afforded by the large area detector combine to produce an exceptionally high count rate for a ToF instrument. Preliminary measurements using the small area detector produce count rates of $5-50 \mathrm{kHz}$. Based on this result, the estimated count rate using the large area detector exceeds $3 \mathrm{MHz}$. Software histogramming and even softwaretriggered data transfer at this rate are not feasible. We have developed a system whereby up to sixteen independent data channels are transferred to CAMAC histogram memory under hardware control. The data acquisition computer pre-sets the ion beam pulse width and pulse repetition rate, and defines the function of each segment of histogram memory. If only the small area detectors are in use, then they may each utilize up to $81 \mathrm{k}$ segments of histogram memory. If the large area detector is in use, it uses $8 \mathrm{lk}$ segments, one for each portion of the divided anode, leaving $41 \mathrm{k}$ segments or 8512 segments for each of the small area detectors.

Once data acquisition has been started, a quartz controlled clock on the data acquisition card controls the pulse repetition rate without further software intervention, and the data is assembled into the requisite number of histograms under hardware control. Consequently, the computer is able control the deposition process and simultaneously assemble composite spectra obtained by the large area detector, display the data, control beam position and build images of the elemental distributions on the surface without disrupting the data acquisition process. Details of the data acquisition hardware will be presented elsewhere. [73]

\subsection{Results}

Preliminary ICISS results have been obtained for metals and HTSC oxide thin films using a $165^{\circ}$ scattering angle with a small area detector for $\mathrm{He}^{+}, \mathrm{Ne}^{+}$and $\mathrm{Ar}^{+}$pulsed beams. The results presented here all represent detection of backscattered ions plus neutrals. $\mathrm{W}, \mathrm{Nb}$, $\mathrm{Cu}$ and $\mathrm{Au}$ metal foils and lava were used as calibration standards, and compared with data obtained from $\mathrm{YBa}_{2} \mathrm{Cu}_{3} \mathrm{O}_{7-\mathrm{x}}$ (YBCO) and $\mathrm{Bi}_{2} \mathrm{Sr}_{2} \mathrm{Ca}_{2} \mathrm{Cu}_{3} \mathrm{O}_{8}$ (BSCCO) HTSC films.

Figure 28 shows ToF spectra for $10 \mathrm{keV} \mathrm{Ar}+$ incident on $\mathrm{Au}$ and $\mathrm{W}$ foil samples. Both samples were ultrasonically degreased prior to mounting, and subjected to a brief ion beam sputter etching before starting analysis, using a dc beam current density of $\sim 2 \times 10^{-5} \mathrm{~A} / \mathrm{cm}^{2}$ obtained from the Atomika ion source. The high velocity side of both peaks is very sharp, and the small calculated difference of $0.14 \mu \mathrm{sec}$ is clearly distinguishable. The W peak however, exiends further toward low velocities as a result of multiple scattering from residual low mass surface contaminants. Lightly oxidized metal surfaces have a high probability of ejecting electrons upon ion impact. which are detected by the electron multiplier detector almost immediately after the primary ion strikes the surface, thereby providing a calibration for the origin of the ToF scale. The sharp peak located at $3.79 \mu \mathrm{sec}$ also corresponds to the calculated time lag between the initiation of the ion beam pulse and its impact at the sample surface. Using this value for $\mathrm{ToF}=0$, the peak positions for $\mathrm{Au}$ and $\mathrm{W}$ are calculated and plotted as the indicated data points. Gold, which does not form an oxide, does not display the sharp peak.

Insulating oxides are potentially a source of difficulty for any charged particle spectroscopy since the surface will charge if the net incident charged particle flux does not match the outgoing flux. In principle, if the surface charges positively, the problem can be remedied through the use of an electron flood gun. However, the ion beam dose for the ToF ISS system is so low that even this remedy may not be necessary. Unless otherwise noted, the data shown in this section were acquired using a $10 \mathrm{keV} \mathrm{He} \mathrm{He}^{+}, \mathrm{Ne}^{+}$or $\mathrm{Ar}^{+}$beam with $50 \mathrm{nsec}$ pulse length and time averaged currents of $2-4 \times 10^{-11}$ amps, with typical spot sizes 200-300 $\mu \mathrm{m}$ in diameter. Data acquisition times ranged from $\sim 4-40$ seconds, resulting in beam doses of roughly $5 \times 10^{11}-5 \times 10^{12}$ ions/cm². Fig. 29 shows a ToF spectrum for $7 \mathrm{keV} \mathrm{Ne}{ }^{+}$incident on a lava insulator used as part of the sample holder. Peaks for $\mathrm{Al} . \mathrm{Si}, \mathrm{Cl}$ and $\mathrm{Cu}$ are clearly 
distinguished although all of the peaks have a low energy tail because a major surface constituent, oxygen, is lighter than the probe beam.

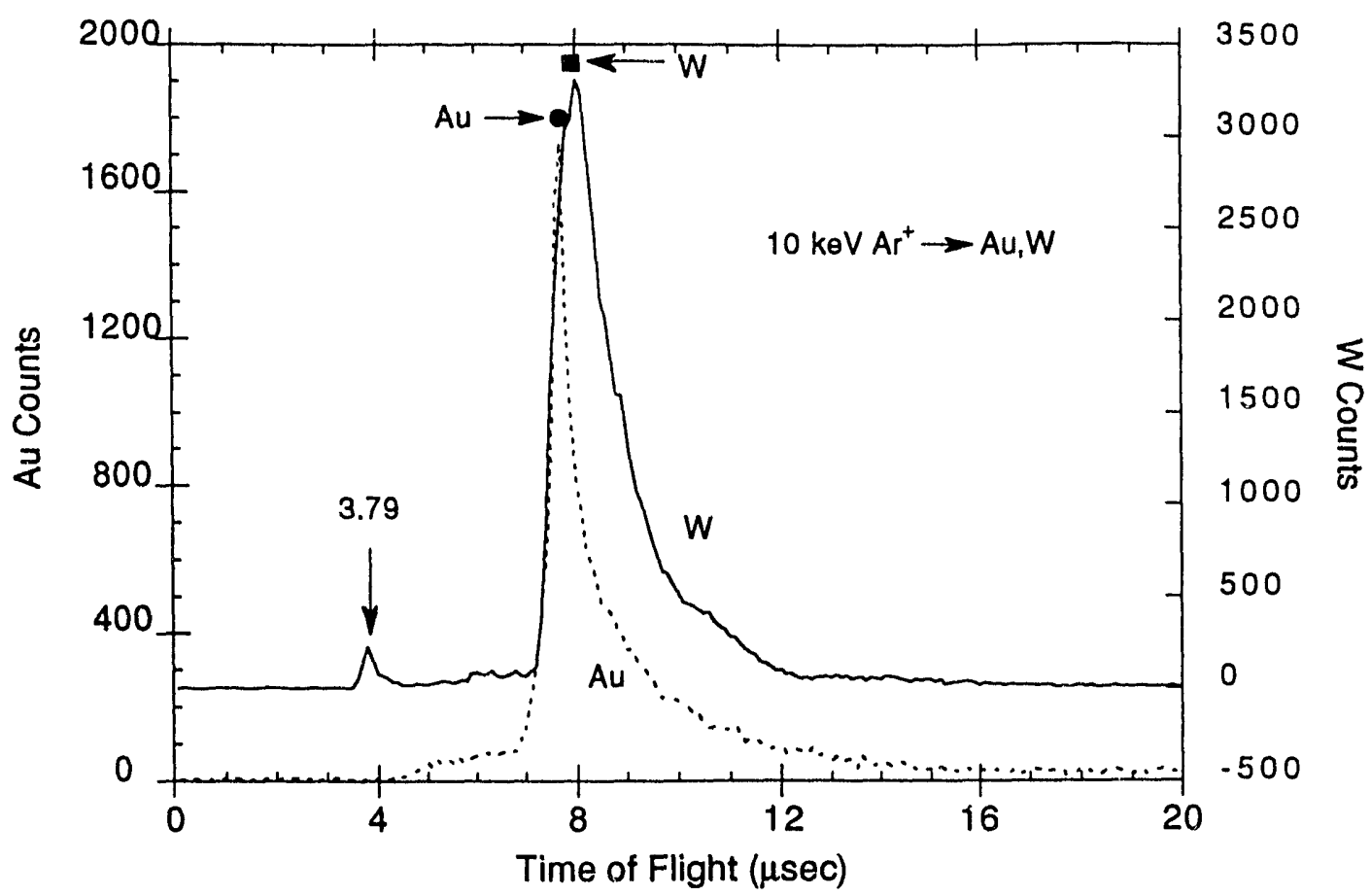

Fig. $28 \mathrm{ToF}$ spectra for $10 \mathrm{keV} \mathrm{Ar}+$ incident on W (solid line) and Au (dashed line). The calculated peak positions are indicated by the symbols.

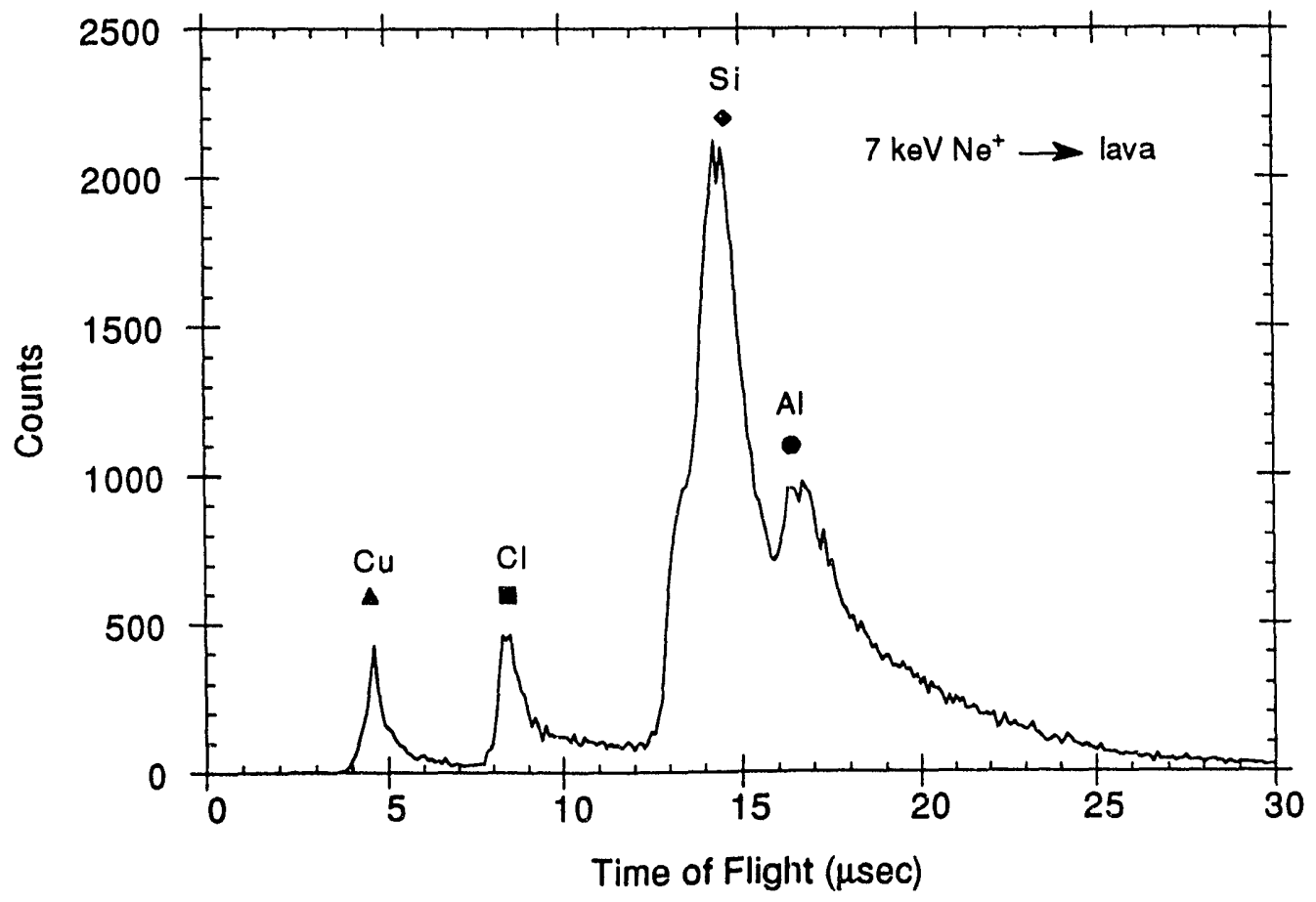

Fig. 29 ToF spectra for $7 \mathrm{keV} \mathrm{Ne}^{+}$incident on lava. The calculated peak positions for $\mathrm{Cu}, \mathrm{Cl}, \mathrm{Si}$ and $\mathrm{Al}$ are indicated by the symbols. 
A ToF spectrum for $10 \mathrm{keV} \mathrm{Ar}^{+}$nomally incident on a BSCCO thin film is shown in Fig. 30. Fig. 30a shows an "as is" surface, exhibiting a broad multiple scattering peak and no clearly resolved elemental lines. The base pressure of the system is in the low $10^{-9}$ torr, and rises to $\sim 4 \times 10^{-8}$ torr when the ion beam is on, although the pressure rise is almost entirely due to the inert gas in the beam. After heating to $150^{\circ} \mathrm{C}$ and sputtering for 34 minutes (Fig. 30b), a strong $\mathrm{Bi}$ line and a weak Sr line were observed. Tuming the beam off for 27 minutes (Fig. 30c) resulted in the disappearance of both elemental lines. The partial pressure of active gas in the chamber is equivalent to the adsorption of 1-2 monolayers during the 27 minute interval. However, rotating the sample so that the beam was incident at a $45^{\circ}$ angle resulted in the reappearance of the Bi signal (Fig. 30d), indicating that the adsorbed gas was in an A-top binding site which shadows the underlying $\mathrm{Bi}$ for normal ion beam incidence.
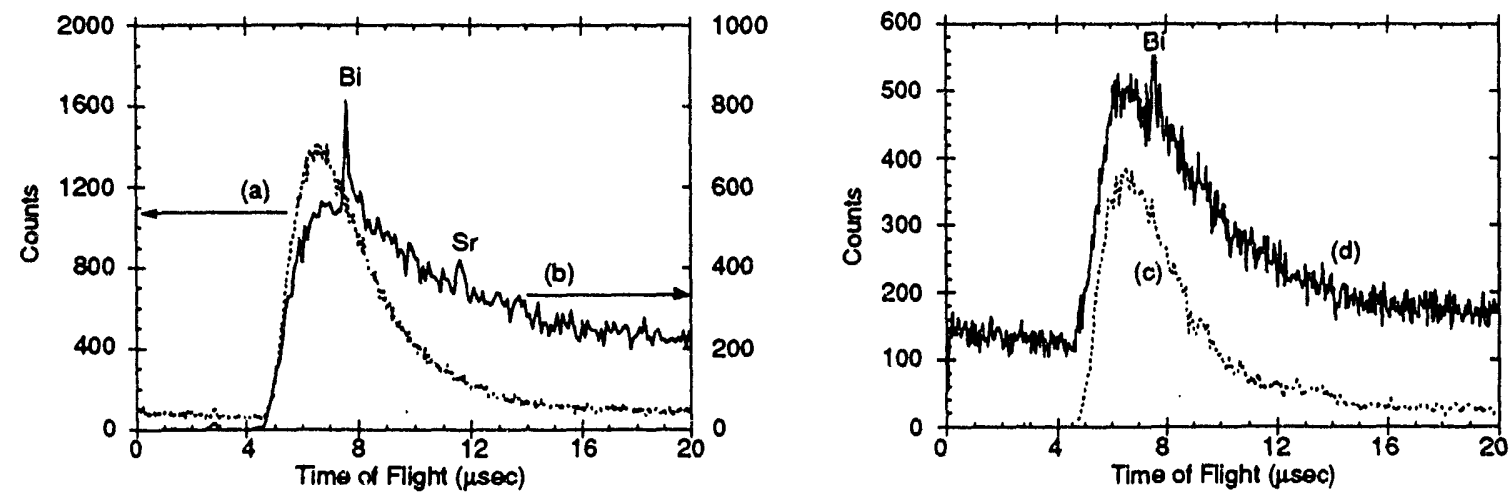

Fig. 30 (a) $10 \mathrm{keV} \mathrm{Ar}{ }^{+}$normal incidence ToF spectrum of an "as is" BSCCO thin film. (b) Same film after sputtering for 34 minutes with $10 \mathrm{keV} \mathrm{Ar}^{+}$(c) same film 27 minutes after sputtering (d) same as $\mathrm{c}$ except for $45^{\circ}$ incidence.

For more extended room temperature sputtering, (Fig. 31a), $\mathrm{Bi}, \mathrm{Sr}$ and $\mathrm{Cu}$ are clearly visible above the background. However, one hour after sputtering ceased, the Sr signal had almost disappeared and the $\mathrm{Cu}$ signal had disappeared completely, while the $\mathrm{Bi}$ signal has not decreased (Fig. 3ib), indicating that adsorption occurs preferentially on the $\mathrm{Sr}$ and $\mathrm{Cu}$ sites as long as they are not covered by more than one monolayer of adsorbate.

Figure 32 represents ToF spectra of a $\mathrm{YBa}_{2} \mathrm{Cu}_{3} \mathrm{O}_{7-x}$ film $240 \AA$ thick in which $\mathrm{Y}, \mathrm{Ba}$ and $\mathrm{Cu}$ were deposited sequentially on a $\mathrm{LaAlO}_{3}$ substrate to produce a film 20 unit cells thick. The inset represents a spectrum taken with $10 \mathrm{a} \mathrm{keV} \mathrm{He}{ }^{+}$beam. which clearly shows the oxygen peak, but is unable to resolve $\mathrm{Y}$ and $\mathrm{Ba}$. The main figure was taken with a $10 \mathrm{keV}$ $\mathrm{Ar}^{+}$beam which clearly resolves $\mathrm{Y}$ and $\mathrm{Ba}$. As $\mathrm{i}_{\mathrm{A}}$ the case with $\mathrm{BSCCO}$, it is very difficult to see the $\mathrm{Cu}$ peak. By subtracting the background, a measure of the surface composition may be obtained. A depth profile is shown in Fig. 33. There is a clear modulation of composition both at the surface and near the interface with the substrate occurring over a distance on the order of $10 \AA$. The half width of the onset of the substrate signal is approximately $4 \AA$, corresponding to $1 / 3$ of the $c$-axis lattice spacing of $\mathrm{YBa}_{2} \mathrm{Cu}_{3} \mathrm{O}_{7-x}$. Depth resolution on this fine a scale is very difficult to obtain via conventional surface analytical methods.

A preliminary angular profile of the $Y$ and Ba signals for the sample of Figs. 32 and 33 is shown in Fig. 34. A previous ISS study [74] using a cylindrical mirror ESA showed copper and oxygen to decrease relative to $\mathrm{Ba}$ during the orthorhombic to tetragonal phase transition. Both the $\mathrm{Cu}-\mathrm{O}$ chains and $\mathrm{Ba}$ were found to be located in the first monolayer. However, issues of beam damage and ion neutralization effects remain to be clarified. The data presented in Fig. 34 show strong $\mathrm{Ba}$ intensity oscillations at near-grazing incidence and strong intensity oscillations in the $Y$ signal near normal incidence, suggesting that $Y$ occupies second layer sites which are offset relative to the surface $\mathrm{Ba}$ atom positions. 


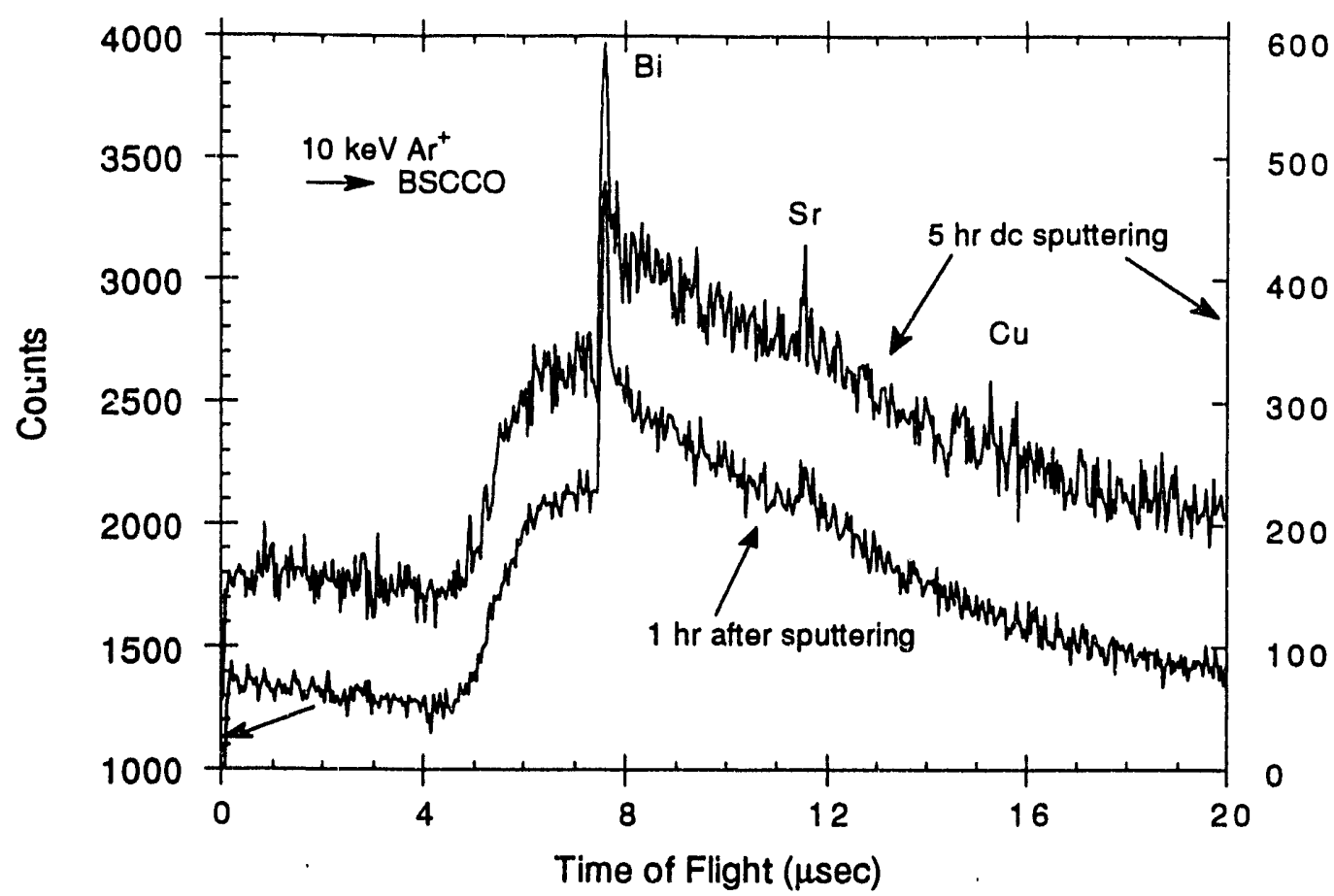

Fig. 31 (a) $10 \mathrm{keV} \mathrm{Ar}{ }^{+}$normal incidence ToF spectrum of a BSCCO thin film after sputtering for 5 hours. (b) same as $4 \mathrm{a}$ one hour after sputtering.

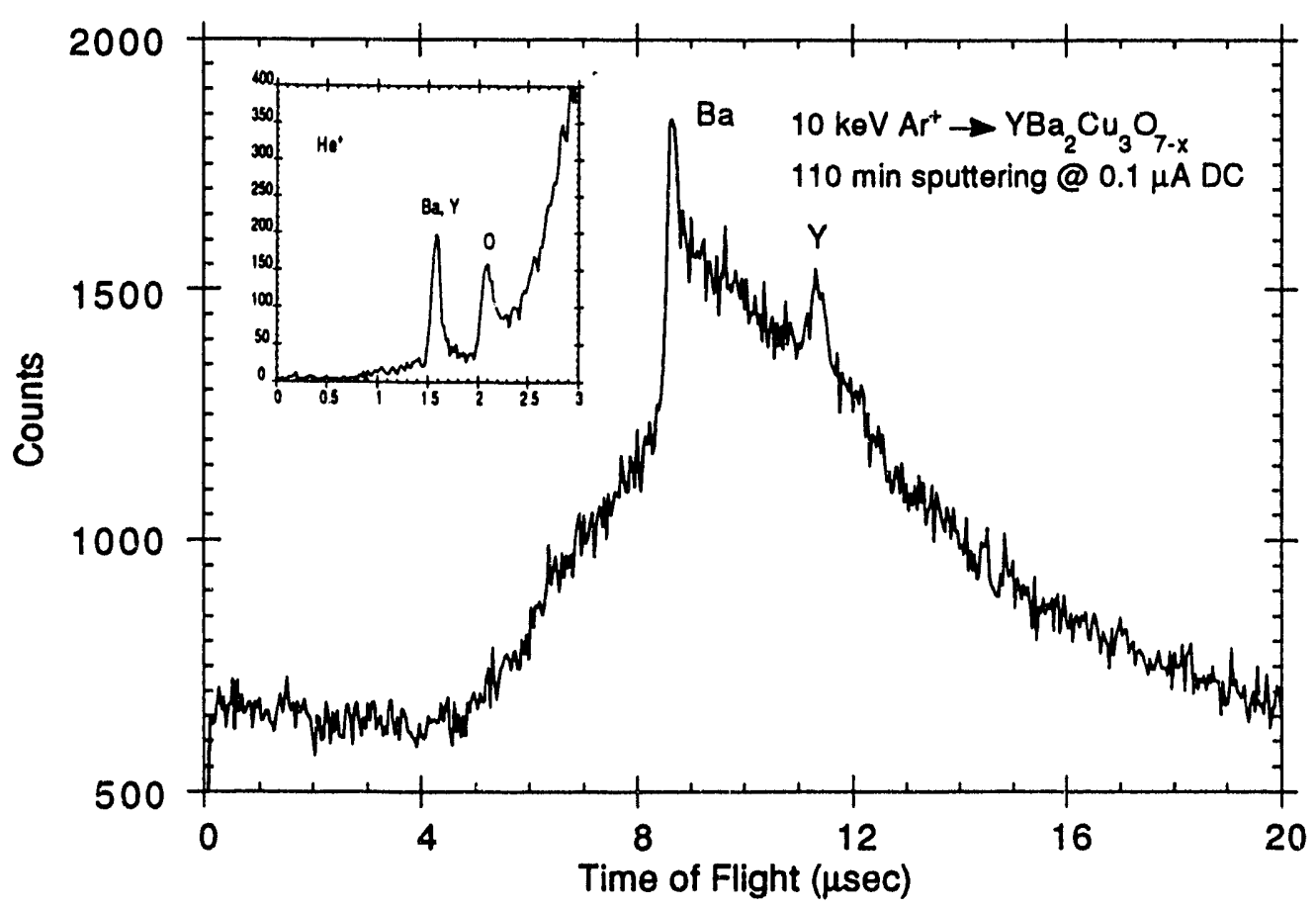

Fig. $3210 \mathrm{keV} \mathrm{He}+$ (inset) and $\mathrm{Ar}^{+}$(main figure) ToF spectra of a $\mathrm{YBa}_{2} \mathrm{Cu}_{3} \mathrm{O}_{7-\mathrm{x}}$ thin film. 


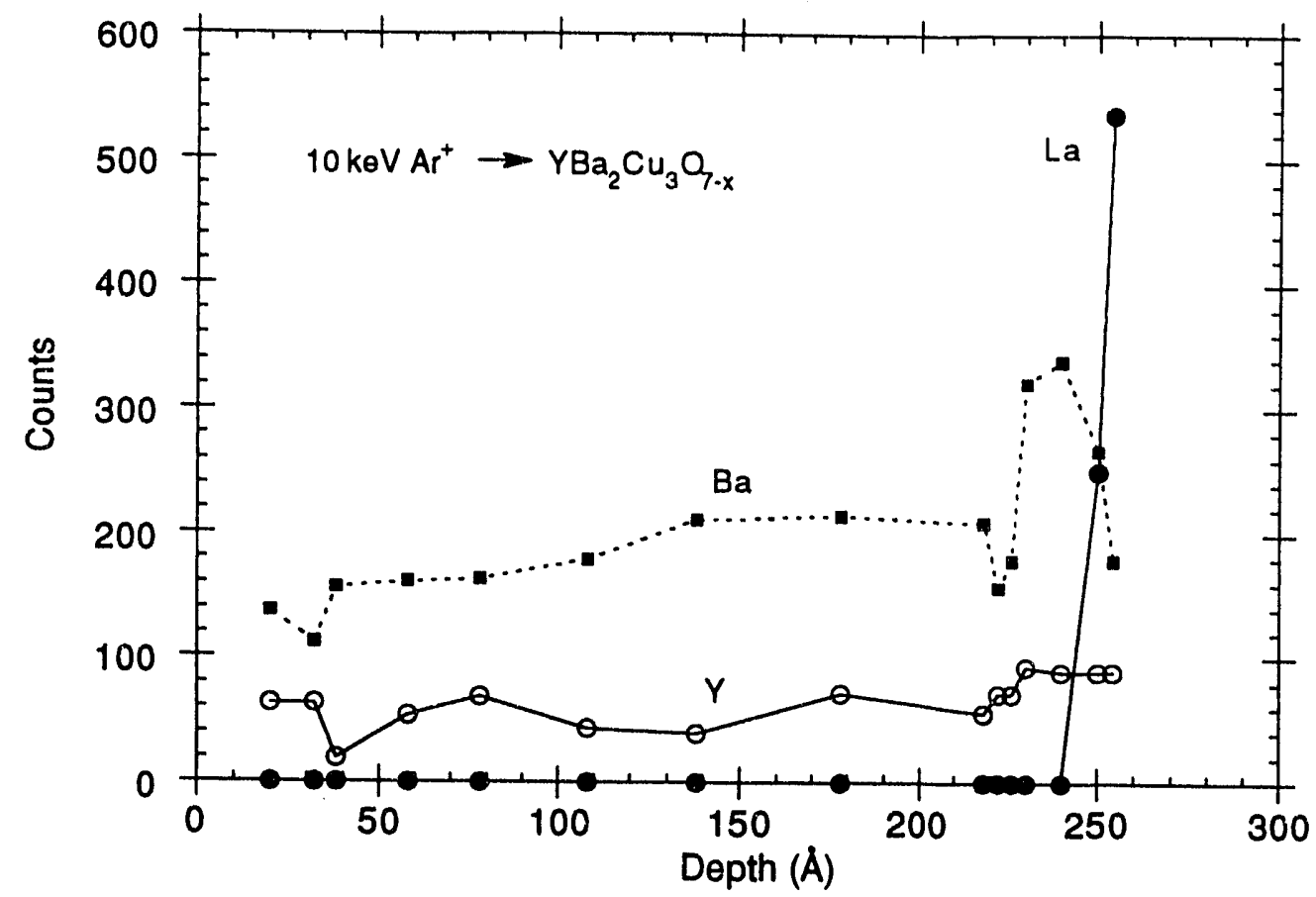

Fig. 33 depth profile of the film in Fig. 32.

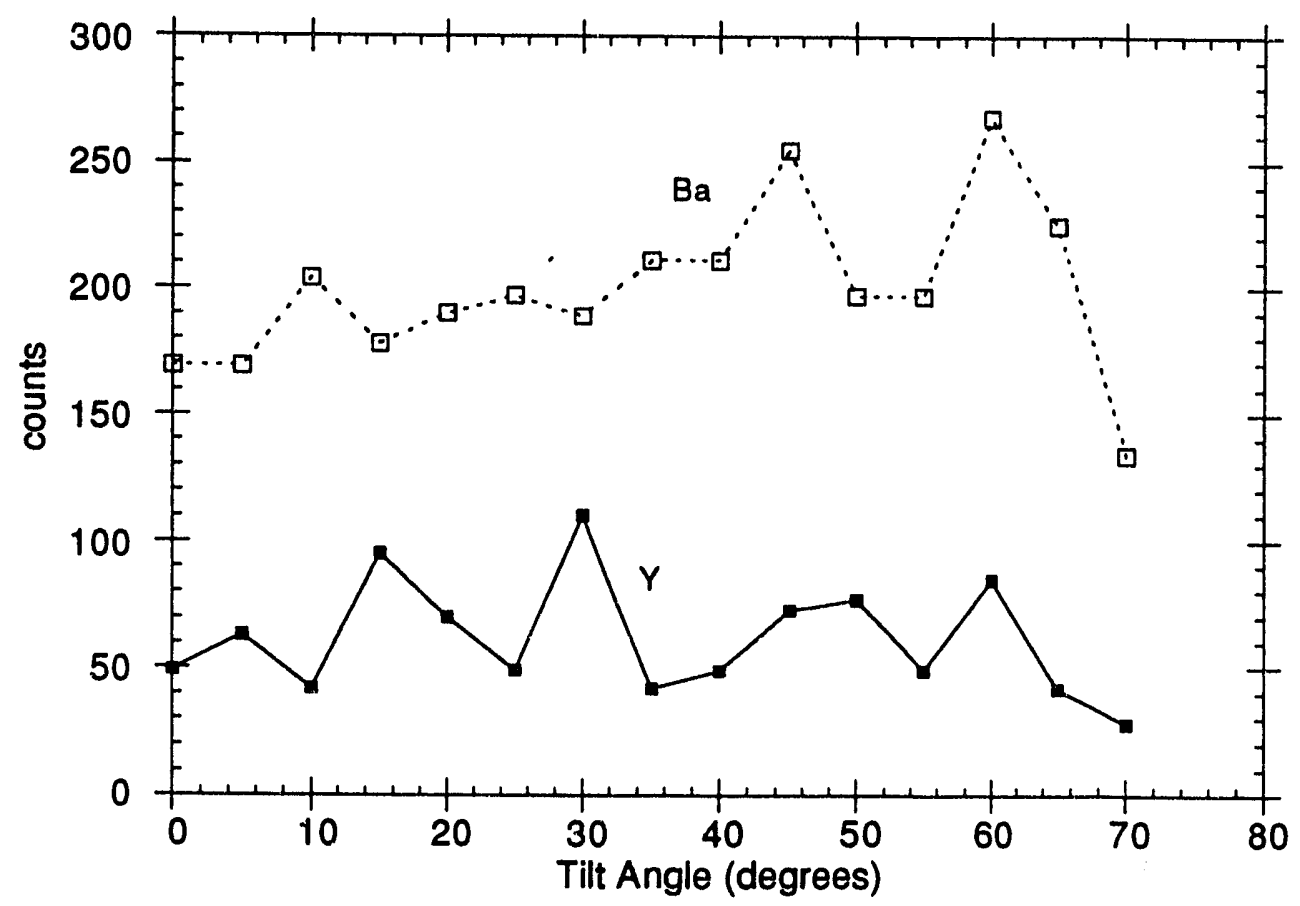

Fig. 34. Polar scan of the $\mathrm{Ba}$ and $\mathrm{Y}$ signal intensities for the film of Fig. 32. 


\subsection{Summary}

Time of Flight pulsed ion beam analysis can provide a wide range of information on the surface characteristics of multicomponent and multilayered thin films. In particular, it is possible to obtain this information at the relatively high chamber pressures required for the growth of oxide and nitride thin films. The instrumentation does not conflict with the spatial constraints of most thin film deposition processes, the required beam dose and consequent beam damage can be extremely low, and data can be collected on a real-time basis. A pulsed ion beam surface analysis instrument for this purpose is under development, and its current performance characteristics and anticipated capabilities are described. Preliminary results on HTSC thin fiims indicate that extremely high depth resolution can be obtained for interface profiling, and that information on the relative positions of the different atomic species in the near-surface region and gas phase adsorption sites may be determined.

\section{Acknowledgement}

This work has been supported in part by the U. S. Department of Energy, Office of Basic Energy Sciences under contract W-31-109-ENG-38 and by the State of Illinois Dept. of Commerce and Community Affairs.

\section{References}

1. O. Auciello, A. I. Kingon, A. R. Krauss, and D. J. Lichtenwalner, Proc. NATO Advanced Study Institute on "Multicomponent and Multilayered Thin Films for Advanced Microtechnologies, Techniques, Fundamentals and Devices" , Bad Windsheim, Fed. Rep. Germany, Sept. 22-Oct. 6, 1992

2. M. Hawley, I. D. Raistrick, J. G. Beery, and R. J. Houlton, Science 251, 279 (1991)

3. D. P. Smith, Surf. Sci. 25, 171 (1971)

4. J.T. McKinney and R.P. Frankenthal, Proc. Annual Meeting National Association of Corrosion Engineers, March 22, 1973, Anaheim, CA.

5. D. P. Smith, J. Appl. Phys. 38, 340 (1967)

6. T. M. Buck, G. H. Wheatley and L. K. Verheij, Surf. Sci. 90, 635 (1979)

7. L. Marchut, T. M. Buck, G. H. Wheatley and C. J. McMahon, Surf. Sci. 141, 549 (1984)

8. R. Goff, J. Vac. Sci. Technol. 10, 355 (1973)

9. J. Lindhard, V. Nielsen, M. Scharff, Mat. Fys. Kongl. Dansk. Vid. Selskab 36 (1968) 1

10. J. Lindhard, M. Scharff, and H. E. Schiott, Mat. Fys. Kongl. Dansk. Vid. Selskab 33, 14 (1968) 1

11. J. Lindhard, V. Nielsen, M. Scharff, and P. V. Thomsen, Mat. Fys. Kongl. Dansk. Vid. Selskab 33,10 (1968) 1

12. G. Moliere, Z. Naturforsch. 2a, 133 (1947)

13. H. D. Hagstrum, Phys. Rev. 96 (1954) 336

14. A. Barcz, M. Croset, L. M. Mercandalli, Surface Science 95, 522 (1980)

15. D. J. Ball, T. M. Buck, D. MacNair and G. H. Wheatley, Surface Science 30, 69 (1972)

16. A. R. Krauss and V. Krohn, "Aspects of Secondary Ion Emission" in Mass Spectrometry Vol. 6 Specialist Periodic Reports, Royal Society of Chemistry, London (1980)

17. M. Grundner, W. Heiland and E. Taglauer, Appl. Phys. 4, 243 (1974)

18. J. W. Rabalais and J. Chen, J. Chem. Phys. 85, 3617 (1986)

19. R. S. Bhattacharya, W. Eckstein and H. Verbeek, Surface Science 93, 563 (1980)

20. R. L. Erickson and D. P. Smith, Phys. Rev. Lett. 34 (1975) 297

21. A. Zartner, E. Taglauer and W. Heiland, Phys. Rev. Lett. 40 (1978) 1259

22. D. P. Woodruff, Nucl. Instrum. Meth. 194 (1982) 639

23. R. C. McCune, J. Vac. Sci. Technol. 18 (1981) 700

24. E. Taglauer, W. Englert, W. Heiland and D. P. Jackson, Phys. Rev. Lett. 45 (1980) 740

25. S. H. Overbury, Surf. Sci. 112 (1981) 23

26. H. Niehus and E. Preuss, Surf. Sci. 119 (1982) 349

27. H. Niehus, Surf. Sci. 145 (1984) 407

28. H. Niehus, and G. Comsa, Nucl. Instrum. Meth. Phys. Res. B15 (1986) 122

29. H. Niehus, J. Vac. Sci. Technol. A5 (1987) 751 
30. H. Niehus and G. Comsa, Surf. Sci. 140 (1984) 18

31. H. Niehus, Surf. Sci. 166 (1986) L. 107

32. H. H. Brongersma, J. Vac. Sci. Technol. 11 (1974) 231

33. W. Heiland and E. Taglauer, Surface Sci. 68 (1977) 96

34. L. K. Verheij, J. A. Van den Berg, and D. G. Armour. Surface Science 84 (1979) 408

35. W. Moritz and D. Wolf, Surface Sci. 88 (1979) L29

36. J. A. Van den Berg, L. K. Verheij, and D. G. Armour, Surface Science 91 (1980) 218

37. R. M. Tromp, J. Vac. Sci. Technol. Al (1983) 1047

38. E. Taglauer, Appl. Phys. A38 (1985) 161

39. R. Margraf, H. Knözinger and E. Taglauer, Surf. Science 211/212 (1989) 1083

40. M. Katayama, E. Nomura, N. Kanekama, H. Soejima, and M. Aono, Nucl. Instr. Meth. Phys. Res. B33, 857 (1988)

41. H. Niehus and R. Spitzl, Surface and Interface Analysis 17 (1991) 287

42. Niehus 1991, Appl. Phys. A53 (1991) 388

43. R. Souda, M. Aono, C. Oshima, S. Otani, Y. Ishizawa, Surf. Sci. Lett. 128 (1983) L236

44. H. Niehus, K. Mann, B. N. Eldridge and M. L. Yu, J. Vac. Sci. Technol. A6 (1988) 625

45. E. van de Riet, J. M. Fluit and A. Niehaus, Vacuum 41 (1990) 372,

46. H. Dürr, R. Schneider and Th. Fauster, Phys. Rev. B43 (1990) 12187

47. H. Dürr, R. Schneider and Th. Fauster, Vacuum 41 (1990) 376

48. G. Engelmann and E. Taglauer, Surf. Sci. 162 (1985) 921].

49. E. van de Riet, J. M. Fluit and A. Niehaus, Vacuum 41 (1990) 372,

50. H. Dürr, R. Schneider and Th. Fauster, Phys. Rev. B43 (1990) 12187

51. H. Dürr, R. Schneider and Th. Fauster, Vacuum 41 (1990) 376

52. G. Engelmann and E. Taglauer, Surf. Sci. 162 (1985) 921].

53. H. H. Brongersma, J. Vac. Sci. Technol. 11, 231 (1974)

54. H. Niehus and G. Comsa, Surf. Sci. 93, L147 (1980)

55. M. Aono, C. Oshima, S. Zaima, S. Otani, Y. Ishizawa, Jpn. J. Appl. Phys. 20 (1981), L829

56. M. Aono, Y. Hou, C. Oshima, and Y. Ishizawa, Phys. Rev. Lett. 49 (1982) 567

57. S. Zaima, Y, Shibata, H. Adachi, C. Oshima, S. Otani, M. Aono and Y. Ishizawa, Surface Science 157 (1984) 380

58. M. Aono, Nucl. Instrum. Meth. B2 (1984) 374

59. M. Aono and R. Souda, Jpn. J. Appl. Phys. 24 (1985) 1249

60. J. Möller, H. Niehus, and W. Heiland, Surface Sci. 166 (1986) L111

61. H. Niehus, J. Vac. Sci. Technol. A5, 751 (1987)

62. M. Aono, Y. Hou, R. Souda, C. Oshina, S. Otani, Y. Ishizawa, K. Matsuda and R. Shimizu, Jpn. J. Appl. Phys. 21 (1982) L670

63. B. V. King, M. Katayama, M. Aono, R. S. Daley and R. S. Williams, Vacuum 41 (1990) 938

64. H. K. Schmidt, J. A. Schultz and Z. Sheng, Proc. NATO ASI, Castelvecchio, Italy, July 22-Aug. 3, 1990

65. A. R. Krauss, A. B. DeWald, P. Scott and H. Savage, Fusion Technol. 19 (1991) 913

66. A. R. Krauss, O. Auciello, A. Uritani, M. Valentine, M. Mendelsohn and D. M. Gruen, Nucl. Instrum. Meth. Phys. Res. B27 (1987) 209

67. M. Aono, M. Katayama, E. Nomura, T. Chasse, D. Choi and M. Kato, Nucl. Instr. Meth. B37, 264 (1989)

68. B. L.Doyle, R. T. McGrath, A. E. Pontau, Nucl. Instrum. Meth. Phys. Res. B22, 34 (1987)

69. A. R. Krauss, M. Rangaswamy, J. A. Schultz, H. Schmidt, G. Lamich and D. M. Gruen Thin Solid Films, in press, 1992

70. A. R. Krauss, O. Auciello, A. I. Kingon, M. S. Ameen, Y. L. Liu, T. Barr, T. M. Graettinger, S. H. Rou, C. S. Soble and D. M. Gruen, Applied Surface Science 46, 67 (1990)

71. W. Eckstein, Nucl. Instrum. Meth. Phys. Res. B27, 78 (1987)

72. A. R. Krauss, M. Rangaswamy, in preparation

73. A. R. Krauss, G. Lamich, in preparation

74. S. Tanaka, T. Nakamura, M. Liyama, N. Yoshida, S. Takano, F. Shoji and K. Oura, Appl. Phys. Lett. 59 (1991) 3637 


$$
\begin{aligned}
& \text { DATE } \\
& \text { FILMED } \\
& \text { OI } 121193
\end{aligned}
$$


